Article

\title{
Qualitative Chemical Characterization and Multidirectional Biological Investigation of Leaves and Bark Extracts of Anogeissus leiocarpus (DC.) Guill. \& Perr. (Combretaceae)
}

\author{
Giustino Orlando ${ }^{1}$, Claudio Ferrante ${ }^{1, *,+} \oplus$, Gokhan Zengin ${ }^{2, *,+}\left(\mathbb{D}\right.$, Kouadio Ibrahime Sinan ${ }^{2}$, \\ Kouadio Bene $^{3}$, Alina Diuzheva ${ }^{4} \mathbb{D}^{\mathbb{0}}$, József Jekő ${ }^{5}$, Zoltán Cziáky ${ }^{5}{ }^{\circledR}$, Simonetta Di Simone ${ }^{1}$, \\ Lucia Recinella ${ }^{1}$, Annalisa Chiavaroli ${ }^{1}$, Sheila Leone ${ }^{1}$, Luigi Brunetti ${ }^{1}$, \\ Carene Marie Nancy Picot-Allain ${ }^{6}$, Mohamad Fawzi Mahomoodally ${ }^{6, *(D)}$ and Luigi Menghini ${ }^{1}$ \\ 1 Department of Pharmacy, University “G. d'Annunzio" of Chieti-Pescara, 66100 Chieti, Italy \\ 2 Department of Biology, Faculty of Science, Selcuk University, Konya 42130, Turkey \\ 3 Laboratoire de Botanique et Phytothérapie, Unité de Formation et de Recherche Sciences de la Nature, \\ Université Nangui Abrogoua, 02 BP 801 Abidjan 02, Ivory Coast \\ 4 Department of Forest Protection and Entomology, Faculty of Forestry and Wood Sciences, Czech University \\ of Life Sciences, 16500 Prague, Czech Republic \\ 5 Agricultural and Molecular Research and Service Institute, University of Nyíregyháza, 4400 Nyíregyháza, \\ Hungary \\ 6 Department of Health Sciences, Faculty of Science, University of Mauritius, 230 Réduit, Mauritius \\ * Correspondence: claudio.ferrante@unich.it (C.F.); gokhanzengin@selcuk.edu.tr (G.Z.); \\ f.mahomoodally@uom.ac.mu (M.F.M.) \\ + These Authors contributed equally to this work.
}

Received: 8 August 2019; Accepted: 22 August 2019; Published: 1 September 2019

Abstract: Anogeissus leiocarpus (DC.) Guill. \& Perr. (Combretaceae) has a long history of use by folk populations for the management of multiple human ailments. Based on the published literature, there has been no attempt to conduct a comparative assessment of the biological activity and the phytochemical profiles of the leaves and stem bark of A. leiocarpus extracted using methanol, ethyl acetate, and water. By high-performance liquid chromatography with electrospray ionization mass spectrometric detection (HPLC-ESI-MSn ${ }^{\text {}}$ analysis, quinic, shikimic, gallic, and protocatechuic acids were tentatively identified from all the extracts, while chlorogenic, caffeic, ferulic, and dodecanedioic acids were only characterised from the leaves extracts. Additionally, a pharmacological study was carried out to evaluate potential protective effects that are induced by the extracts in rat colon and colon cancer HCT116 cell line. In general, the methanol and water extracts of $A$. leiocarpus leaves and stem bark showed potent radical scavenging and reducing properties. It was noted that the stem bark extracts were more potent antioxidants as compared to the leaves extracts. The methanol extract of $A$. leiocarpus leaves showed the highest acetyl (4.68 mg galantamine equivalent/g) and butyryl (4.0 mg galantamine equivalent/g) cholinesterase inhibition. Among ethyl acetate extracts, the pharmacological investigation suggested stem bark ethyl acetate extracts to be the most promising. This extract revealed ability to protect rat colon from lipopolysaccharide-induced oxidative stress, without exerting promoting effects on HCT116 cell line viability and migration. As a conclusion, A. leiocarpus represents a potential source of bioactive compounds in the development of novel therapeutic agents.

Keywords: Anogeissus; bioactive compounds; antioxidant; enzyme inhibition; ulcerative colitis 


\section{Introduction}

Anogeissus leiocarpus (DC.) Guill. \& Perr. (Combretaceae), also known as chewing stick or axlewood tree, has a long history of traditional use for the management of multiple human ailments. The leaves of $A$. leiocarpus are used in the treatment of skin diseases, fever, diarrhoea, malaria, and stomach infections [1]. A. leiocarpus is used by the Yoruba people in Nigeria to treat bacterial infections and the roots and twigs of the plant are used as chewing sticks for dental hygiene. Various parts of the plant (roots, leaves, stem bark, and twigs) are used in the management of gonorrhoea, cough, wounds, acute respiratory tract infections, stomach infections, fever, tuberculosis, dysentery, giardiasis, malaria, trypanosomiasis, yellow fever, jaundice, and pathogenic microbial infections [2]. The water extract of $A$. leiocarpus stem bark was recently found to combat erectile dysfunction in paroxetine-induced sexually impaired male Wistar rats [3]. A spontaneous decrease in serum glucose level in alloxan-induced diabetic rats administered with the aqueous extract of $A$. leiocarpus leaves [4] was linked to the $\alpha$-amylase and $\alpha$-glucosidase inhibitory action of the extract [5]. The aqueous extract of A. leiocarpus trunk bark was reported to exert significant antihypertensive effects in NG-nitro-L-arginine methyl ester (L-NAME)-induced hypertensive rats [6]. The methanolic and ethyl acetate extracts of A. leiocarpus leaves exhibited antioxidant and antibacterial properties [7]. The stem bark methanolic extract of A. leiocarpus demonstrated antitrypanosomal activity against four Trypanosoma strains [8] and leishmanicidal activity [9]. The methylene chloride extract of $A$. leiocarpus ( $\mathrm{IC}_{50}$ value of $3.8 \mu \mathrm{g} / \mathrm{mL}$ ) showed in vitro antiplasmodial activity against Plasmodium falciparum, the protozoan parasite that is responsible for malaria in human [10]. Lately, a group of researchers investigated the effect of A. leiocarpus methanolic extract on the liver function in mice that were infected with Plasmodium berghei [11].

From the literature, several studies attempted to investigate the biological activity, mainly, the antibacterial properties, of different extracts of $A$. leiocarpus. However, as far as our literature search could ascertain, no study was focused on the comparative evaluation of the phytochemical profiles of the methanol, ethyl acetate, and water extracts of the leaves and stem bark of A. leiocarpus. Additionally, in the present study, the authors present the antioxidant and inhibitory action of A. leiocarpus extracts on key enzymes that are related to diabetes type II, Alzheimer's disease, and skin hyperpigmentation. Finally, while considering the potential antiproliferative effects that are exerted by A. latofolia on colon cancer cells [12], the antiproliferative effects of $A$. leiocarpus extracts were tested on human colon cancer HCT116 cell line. Additionally, the same extracts were tested for their putative antioxidant/anti-inflammatory effects on isolated rat colon specimens that were exposed to E. coli lipopolysaccharide (LPS), in order to reproduce the burden of oxidative stress and inflammation occurring in ulcerative colitis [13]. To this regard, selected biomarkers of oxidative stress/inflammation, including prostaglandin $(\mathrm{PG}) \mathrm{E}_{2}, 8$-iso- $\mathrm{PGF}_{2 \alpha}$, and serotonin $(5-\mathrm{HT})$ were selected. It is expected that detailed phytochemical profiles of the different extracts will enable tentative identification of phytochemical/s, which might be responsible for the observed biological activity.

\section{Materials and Methods}

\subsection{Plant Material and Preparation of Extracts}

The sampling of the plant species was done in Gontougo region (Sandegue) of Ivory Coast in the year 2018. Botanical authentication of the plant was done by the botanist Dr. Kouadio Bene (Laboratoire de Botanique et Phytothérapie, Université Nangui Abrogoua, Abidjan, Ivory Coast). The leaves and stem barks were dried at room temperature (in shade, about 10 days). These materials were then powdered by using a laboratory mill.

Methanol and ethyl acetate extracts were prepared through maceration techniques (five grams of plant samples were mixed with one hundred $\mathrm{ml}$ of each solvents for $24 \mathrm{~h}$ ). After maceration, the extracts were subjected to filtration and evaporation in vacuo at $40{ }^{\circ} \mathrm{C}$. Traditional infusion was selected to prepare the water extract (five grams of plant samples were infused with one hundred $\mathrm{mL}$ of boiling 
water for $20 \mathrm{~min}$.). After preparation, the water extract was subjected to filtration and freeze drying. Finally, the extracts were stored at $4{ }^{\circ} \mathrm{C}$ until phytochemical and pharmacological analysis.

\subsection{Profile of Bioactive Compounds}

Total phenols, flavonoids, phenolic acids, and flavonols were assayed through spectrophotometric assays [14,15], The extract concentrations of phenolics, flavonoids, phenolic acids, flavonols and tannins, and saponins were determined through spectrophotometric assays [14,15], and were expressed as equivalents of gallic acid (mg GAE/g dry extract), rutin (mg RE/g dry extract), caffeic acid (mg CAE/g dry extract), catechin (mg CE/g dry extract), and quillaja (mg QE/g dry extract), respectively.

The qualitative analysis of $A$. santonicum extracts $(5 \mathrm{mg} / \mathrm{mL})$ was carried out according the protocol that was described by Zengin et al. [16].

An high performance liquid chromatography (HPLC)-fluorimetric analysis was carried out in order to quantify the selected phenolic compounds, in A. santonicum extracts $(5 \mu \mathrm{g} / \mathrm{mL})$. To this regard, an HPLC apparatus (MOD. 1525, Waters Corporation, Milford, MA, USA) coupled to fluorimetric detector (MOD. 2475, Waters Corporation, Milford, MA, USA) and a C18 reversed-phase column (Phenomenex Kinetex, Torrance, CA, USA, $150 \mathrm{~mm} \times 4.6 \mathrm{~mm}$ i.d., $2.6 \mu \mathrm{m}$ ) were used. The HPLC gradient conditions were selected, as previously mentioned by Rodriguez-Delgado and coworkers [17]. In agreement with the same authors, $\lambda \mathrm{ex}=278 \mathrm{~nm}$ and $\lambda \mathrm{em}=360 \mathrm{~nm}$ were selected in order to analyze the following phenolic compounds: gallic acid, catechin, and epicatechin.

\subsection{Determination of Antioxidant and Enzyme Inhibitory Effects}

The evaluation of anti- $\alpha$-amylase, anti- $\alpha$-glucosidase, anti-cholinesterases, and anti-tyrosinase activities was carried out as previously described by Uysal and coworkers [18]. The enzyme inhibitory results were evaluated in terms of standard equivalents; galatamine for cholinesterase (mg GALAE/g dry extract); kojic acid for tyrosinase (mg KAE/g dry extract), acarbose for amylase, and glucosidase (mmol ACAE/g dry extract). According to the same paper [18], the antiradical activity of the extracts was measured through the use of ferric reducing antioxidant power (FRAP), 2,2'-azino-bis(3-ethylbenzothiazoline-6-sulphonic acid) (ABTS) cupric reducing antioxidant capacity (CUPRAC), 2,2-diphenyl-1-picrylhydrazyl (DPPH), phosphomolydenum, and metal chelating tests. The antioxidant results were explained as equivalents of trolox ( $\mathrm{mg} \mathrm{TE} / \mathrm{g}$ dry extract) and ethylenediaminetetraacetic acid (EDTA) (in metal chelating assay) (mg EDTAE/g dry extract). One-way ANOVA, followed by Tukey's post hoc test, were applied for comparing the samples in terms of bioactive compounds content and biological activities. The MCA (multiple correspondence analysis) and Clustering Image Map were performed for the discrimination between the samples based on their chemical compositions and Venn graph was built to identify the chemical profile differences among those samples. Before, the data of the chemical composition were attributed to classes with two modalities (e.g., + for presence and - for the absence of compounds in extracts). Afterwards, Multiple datasets supervised analysis, namely DIABLO, was achieved to find out the key factor (parts and solvents) that is responsible for variation in datasets. Subsequently, the correlation between the bioactive compounds and biological activities were estimated. All of the statistical tests were conducted by using R 3.5.1 software environment.

\subsection{Pharmacological Assays}

\subsubsection{Allelopathy Assay}

As previously described [19], the allelopathy bioassay was carried out in $90 \mathrm{~mm}$ diameter Petri dishes, which represented the substrate for the germination of seeds, whereas A. leiocarpus extracts $(0.1-10 \mathrm{mg} / \mathrm{mL})$ were dissolved in imbibition water. During the incubation period (three days at $4{ }^{\circ} \mathrm{C}$ ), seeds were monitored in order to evaluate their uniform size and integrity. To this regard, lettuce could be considered as one of the most suitable dicotyledon for allelopathy assay. This is due to both 
fast germination rate and high sensitivity. After the third day of treatment, a root length $\geq 1 \mathrm{~mm}$ was the condition to consider positive the germination of seeds [19]. The experiments were carried out in triplicate and means \pm SEM were determined through the use of GraphPad Prism software (version 5.01).

\subsubsection{Brine Shrimp Lethality Assay}

Artemia salina lethality bioassay was performed, as previously reported [13]. The larvae of brine shrimps were exposed to the extracts $(0.01-10 \mathrm{mg} / \mathrm{mL})$ at $25-28^{\circ} \mathrm{C}$ for $24 \mathrm{~h}$. At the end of the incubation period, brine shrimp lethality was evaluated with the equation $((T-S) / T) \times 100$, being $T$ and $S$ the total number of larvae that were exposed to extracts and living nauplii, respectively. The experiments were carried out in triplicate.

\subsection{In Vitro Studies}

HCT116 cell line (ATCC ${ }^{\circledR}$ CCL-247 ${ }^{\mathrm{TM}}$ ) culture and differentiation were carried out as previously described in our published paper [13]. To evaluate the biocompatibility of A. leiocarpus extracts $(0.1 \mathrm{mg} / \mathrm{mL})$, the 3-(4,5-dimethylthiazol-2-yl)-2,5-diphenyltetrazolium bromide (MTT) viability test was carried out, as recently described [13]. The effects of extracts $(0.1 \mathrm{mg} / \mathrm{mL})$ on HCT116 cell viability was evaluated in comparison to the untreated control group $24 \mathrm{~h}$ after treatment. Finally, the effects of extracts on HCT116 cell spontaneous migration, through the use of wound healing test, as recently reported. Briefly, the cells were challenged with A. leiocarpus extracts $(0.1 \mathrm{mg} / \mathrm{mL})$, and spontaneous migration was monitored at different time points $(0$ and $24 \mathrm{~h})$. The Image-J software (NIH) was used to quantify the scratch area, whereas GraphPad software was employed to calculate mean data at 0 and $24 \mathrm{~h}$ and express them as percentage variation with reference to relative $100 \%$ of that at $0 \mathrm{~h}$.

\subsection{Ex Vivo Studies}

Male adult Sprague-Dawley rats (200-250 g) were sacrificed by $\mathrm{CO}_{2}$ inhalation, and colon specimens were immediately stimulated with Escherichia coli lipopolysaccharide (LPS) $10 \mu \mathrm{g} / \mathrm{mL}$ for $4 \mathrm{~h}$ (incubation period), as recently described [13]. Italian Health Minstry (authorization N. F4738.N.XTQ, delivered on 11th Novembre 2018) approved the experimental procedures.

During the incubation period, the colon specimens were also treated with A. leiocarpus extracts $(0.1 \mathrm{mg} / \mathrm{mL})$. Subsequently, extraction and chromatographic quantification of 5-HT (ng/mg wet tissue) was carried out in colon homogenate, as previously reported [20,21]. Additionally, colon homogenate was assayed for measuring $\mathrm{PGE}_{2}$ and 8-iso- $\mathrm{PGF}_{2 \alpha}$ via radioimmunoassay [22,23].

\subsection{Statistical Analysis}

Data were means \pm SEM and analyzed by one-way analysis of variance (ANOVA), followed by Newman-Keuls post hoc test (GraphPad Prism version 5.01 for Windows, GraphPad Software, San Diego, CA, USA). The data were considered to be significant for $p$ values less than 0.05 . With the aim to apply 3Rs (Reduction, Refinement and Reduction) approach to the ex vivo procedures, the number of animals was determined through the "Resource Equation" $N=(E+T) / T(10 \leq E \leq 20)$ [24]), where $\mathrm{N}, \mathrm{T}$, and $\mathrm{E}$ represent the number of animals, pharmacological treatments, and degree freedom in ANOVA, respectively. 


\section{Results}

\subsection{Phytochemical Profile}

Table 1 presents phytochemical evaluations of the different extracts of $A$. leiocarpus leaves and stem bark. Quantitative determination showed that the stem barks extracts of $A$. leiocarpus (water extract $=$ methanol extract $>$ ethyl acetate extract) possessed significant amounts of phenolics when compared to their respective leaves extracts. The water extract of $A$. leiocarpus leaves showed the highest flavonoid (89.0 mg RE/g) and phenolic acid (14 mg CAE/g) contents. Highest tannin (77.0 mg CE/g), flavanol $(79 \mathrm{mg} \mathrm{CE} / \mathrm{g})$, and saponin $(438 \mathrm{mg} \mathrm{QE} / \mathrm{g}$ ) contents were recorded from the methanol extract of A. leiocarpus leaves. Phenolic acids, such as, protocatechuic acid, chlorogenic acid, caffeic acid, and ferulic acid, were previously reported to be soluble in polar protic solvents, like methanol, except gallic acid, which was readily soluble in water [25]. From Table 1, it is noted that no phenolic acid was recorded in the ethyl acetate extracts of $A$. leiocarpus leaves and stem bark. Caffeic acid, a phenolic acid, was found to be minimally soluble in ethyl acetate [26].

HPLC-fluorimeter analysis that was performed on selected phenolic compounds (i.e., gallic acid, catechin and epicatechin), revealed that leaf extract could have, overall, a higher content of these metabolites than stem bark extract (Table 2). This is consistent with the observed content of the total phenolic acids and flavanols.

Detailed analysis of the chemical composition of the ethyl acetate, methanol, and water extracts of A. leiocarpus leaves and stem bark by HPLC-ESI-MS ${ }^{n}$, while using both positive and negative ionisation modes, was also conducted. The detailed results are given as supplemental materials (Tables S1-S6). The results are also summarized in Table 3. 
Table 1. Quantitative phytochemical determinations of A. leiocarpus leaves and stem bark extracts.

\begin{tabular}{|c|c|c|c|c|c|c|}
\hline Samples & $\begin{array}{c}\text { Total Phenolic Content } \\
\text { (mg GAE/g) }\end{array}$ & $\begin{array}{l}\text { Total Flavonoid Content } \\
\text { (mg RE/g) }\end{array}$ & $\begin{array}{l}\text { Total Flavonol } \\
\text { (mg CE/g) }\end{array}$ & $\begin{array}{l}\text { Total Phenolic acid } \\
\text { (mg CAE/g) }\end{array}$ & $\begin{array}{l}\text { Total Tannin Content } \\
\text { (mg CE/g) }\end{array}$ & $\begin{array}{l}\text { Total Saponin Content } \\
(\mathrm{mgQE} / \mathrm{g})\end{array}$ \\
\hline Leaves-EA & $49 \pm 1^{\mathrm{e}}$ & $35.0 \pm 0.6^{c}$ & $6.0 \pm 0.1^{\mathrm{d}}$ & nd & $6.0 \pm 0.4^{\mathrm{e}}$ & $190 \pm 17^{\mathrm{bc}}$ \\
\hline Leaves-MeOH & $223 \pm 2^{c}$ & $54.0 \pm 0.6^{\mathrm{b}}$ & $79 \pm 3^{\mathrm{a}}$ & $8 \pm 1^{b}$ & $77.0 \pm 0.7^{\mathrm{a}}$ & $438 \pm 54^{\mathrm{a}}$ \\
\hline Leaves-Water & $257 \pm 3^{b}$ & $89.0 \pm 0.2^{\mathrm{a}}$ & $3.46 \pm 0.02^{\mathrm{e}}$ & $14 \pm 1^{\mathrm{a}}$ & $18 \pm 4^{\mathrm{c}}$ & $200 \pm 30^{b c}$ \\
\hline Stem barks-EA & $207 \pm 2^{d}$ & $16 \pm 0.3^{\mathrm{f}}$ & $14.0 \pm 0.3^{c}$ & nd & $17 \pm 0.5^{\mathrm{c}}$ & $171 \pm 24^{\mathrm{bc}}$ \\
\hline Stem barks-MeOH & $271 \pm 1^{\mathrm{a}}$ & $27.0 \pm 0.3^{\mathrm{e}}$ & $28.0 \pm 0.4^{\mathrm{b}}$ & nd & $33 \pm 1^{\mathrm{b}}$ & $230 \pm 34^{b}$ \\
\hline Stem barks-Water & $274 \pm 2^{\mathrm{a}}$ & $33.0 \pm 0.2^{\mathrm{d}}$ & $2.09 \pm 0.01^{\mathrm{e}}$ & $7.0 \pm 0.6^{c}$ & $10.0 \pm 0.1^{\mathrm{d}}$ & $163 \pm 28^{c}$ \\
\hline
\end{tabular}

Values expressed are means \pm SD of three parallel measurements. GAE: Gallic acid equivalent; RE: Rutin equivalent. CE: Catechin equivalent; CAE: Caffeic acid equivalent; QE: Quillaja equivalent. nd: not detected. Superscripts in the same column indicate significant difference in the tested extracts $(p<0.05)$.

Table 2. Phenol content of A. leiocarpus leaves and stem bark ethyl acetate (EA), methanol (MeOH) and water extracts.

\begin{tabular}{lcccccc}
\hline \multicolumn{1}{c}{ Phenolic Compound } & Leaves-EA $\mathbf{~ m g} / \mathbf{g}$ & Leaves-MeOH $\mathbf{~ m g} / \mathbf{g}$ & Leaves-Water Extract $\mathbf{~ m g} / \mathbf{g}$ & Stem Barks-EA mg/g & Stem Barks MeOH mg/g & Stem Barks-Water $\mathbf{~ m g / g}$ \\
\hline Gallic acid & $226 \pm 21$ & $89 \pm 28$ & $30 \pm 3$ & $65 \pm 6$ & $38 \pm 2$ & $37 \pm 1$ \\
Catechin & $7.0 \pm 0.8$ & $3.0 \pm 0.2$ & $9 \pm 1$ & $1.0 \pm 0.1$ & $0.29 \pm 0.02$ \\
Epicatechin & $0.29 \pm 0.02$ & $2.0 \pm 0.1$ & $0.27 \pm 0.02$ & $0.27 \pm 0.02$ & $0.18 \pm 0.01$ \\
\hline
\end{tabular}


Table 3. Chemical composition of A. leiocarpus extracts.

\begin{tabular}{|c|c|c|c|c|c|c|c|c|c|c|}
\hline No. & Name & Formula & {$[\mathrm{M}+\mathrm{H}]^{+}$} & {$[\mathbf{M}-\mathbf{H}]^{-}$} & Leaves-EA & Leaves-MeOH & Leaves-Water & $\begin{array}{c}\text { Stem } \\
\text { Bark-EA }\end{array}$ & $\begin{array}{c}\text { Stem } \\
\text { Bark-MeOH }\end{array}$ & $\begin{array}{c}\text { Stem } \\
\text { Bark-Water }\end{array}$ \\
\hline 1 & Quinic acid & $\mathrm{C}_{7} \mathrm{H}_{12} \mathrm{O}_{6}$ & & $19,105,557$ & + & + & + & + & + & + \\
\hline 2 & Hexahydroxydiphenoylhexose & $\mathrm{C}_{20} \mathrm{H}_{18} \mathrm{O}_{14}$ & & $48,106,184$ & - & - & - & + & + & + \\
\hline 3 & Shikimic acid & $\mathrm{C}_{7} \mathrm{H}_{10} \mathrm{O}_{5}$ & & $17,304,500$ & + & + & + & + & + & + \\
\hline 4 & Galloylquinic acid isomer 1 & $\mathrm{C}_{14} \mathrm{H}_{16} \mathrm{O}_{10}$ & & $34,306,653$ & - & - & - & - & + & + \\
\hline 5 & Galloylhexose isomer 1 & $\mathrm{C}_{13} \mathrm{H}_{16} \mathrm{O}_{10}$ & & $33,106,653$ & - & - & - & + & + & + \\
\hline 6 & Galloylhexose isomer 2 & $\mathrm{C}_{13} \mathrm{H}_{16} \mathrm{O}_{10}$ & & $33,106,653$ & - & - & - & + & + & + \\
\hline $7^{1}$ & $\begin{array}{c}\text { Gallic acid } \\
(3,4,5-\text { Trihydroxybenzoic acid })\end{array}$ & $\mathrm{C}_{7} \mathrm{H}_{6} \mathrm{O}_{5}$ & & $16,901,370$ & + & + & + & + & + & + \\
\hline 8 & Galloylhexose isomer 3 & $\mathrm{C}_{13} \mathrm{H}_{16} \mathrm{O}_{10}$ & & $33,106,653$ & - & - & - & + & + & + \\
\hline 9 & Galloylquinic acid isomer 2 & $\mathrm{C}_{14} \mathrm{H}_{16} \mathrm{O}_{10}$ & & $34,306,653$ & - & - & - & - & + & + \\
\hline 10 & Galloylquinic acid isomer 3 & $\mathrm{C}_{14} \mathrm{H}_{16} \mathrm{O}_{10}$ & & $34,306,653$ & - & - & - & - & + & + \\
\hline 11 & Gallocatechin & $\mathrm{C}_{15} \mathrm{H}_{4} \mathrm{O}_{7}$ & & $30,506,613$ & - & + & + & + & + & + \\
\hline 12 & $\begin{array}{c}\text { Protocatechuic acid } \\
\text { (3,4-Dihydroxybenzoic acid) }\end{array}$ & $\mathrm{C}_{7} \mathrm{H}_{6} \mathrm{O}_{4}$ & & $15,301,879$ & + & + & + & + & + & + \\
\hline 13 & 3-Hydroxybenzaldehyde & $\mathrm{C}_{7} \mathrm{H}_{6} \mathrm{O}_{2}$ & $12,304,461$ & & + & + & + & - & - & - \\
\hline 14 & Procyanidin B isomer 1 & $\mathrm{C}_{30} \mathrm{H}_{26} \mathrm{O}_{12}$ & & $57,713,460$ & - & - & - & + & + & + \\
\hline 15 & Punicalagin & $\mathrm{C}_{48} \mathrm{H}_{28} \mathrm{O}_{30}$ & & $108,305,872$ & + & + & + & + & + & + \\
\hline 16 & Kynurenic acid & $\mathrm{C}_{10} \mathrm{H}_{7} \mathrm{NO}_{3}$ & $19,005,042$ & & - & + & + & - & - & - \\
\hline 17 & Procyanidin B isomer 2 & $\mathrm{C}_{30} \mathrm{H}_{26} \mathrm{O}_{12}$ & & $57,713,460$ & - & - & - & + & + & + \\
\hline $18^{1}$ & Catechin & $\mathrm{C}_{15} \mathrm{H}_{14} \mathrm{O}_{6}$ & & $28,907,121$ & + & + & + & + & + & + \\
\hline $19^{1}$ & Epigallocatechin & $\mathrm{C}_{15} \mathrm{H}_{14} \mathrm{O}_{7}$ & & $30,506,613$ & - & + & + & + & + & + \\
\hline 20 & Casuarinin & $\mathrm{C}_{41} \mathrm{H}_{28} \mathrm{O}_{26}$ & & $93,507,906$ & + & + & + & + & + & + \\
\hline 21 & $\begin{array}{c}\text { Chlorogenic acid } \\
\text { (3-O-Caffeoylquinic acid) }\end{array}$ & $\mathrm{C}_{16} \mathrm{H}_{18} \mathrm{O}_{9}$ & $35,510,291$ & & + & + & + & - & - & - \\
\hline 22 & Caffeic acid & $\mathrm{C}_{9} \mathrm{H}_{8} \mathrm{O}_{4}$ & & $17,903,444$ & + & + & + & + & + & + \\
\hline 23 & Cornusiin B or isomer & $\mathrm{C}_{48} \mathrm{H}_{30} \mathrm{O}_{30}$ & & $108,507,437$ & + & + & + & + & + & - \\
\hline 24 & Ampelopsin (Dihydromyricetin) & $\mathrm{C}_{15} \mathrm{H}_{12} \mathrm{O}_{8}$ & & $31,904,540$ & + & + & + & + & + & + \\
\hline 25 & Tellimagrandin I or isomer & $\mathrm{C}_{34} \mathrm{H}_{26} \mathrm{O}_{22}$ & & $78,508,375$ & + & + & + & + & + & + \\
\hline 26 & Coumaroylquinic acid & $\mathrm{C}_{16} \mathrm{H}_{18} \mathrm{O}_{8}$ & & $33,709,235$ & + & + & + & - & - & - \\
\hline 27 & Corilagin or isomer & $\mathrm{C}_{27} \mathrm{H}_{22} \mathrm{O}_{18}$ & & $63,307,279$ & + & + & + & - & - & - \\
\hline 28 & Caffeoylshikimic acid & $\mathrm{C}_{16} \mathrm{H}_{16} \mathrm{O}_{8}$ & & $33,507,670$ & - & + & + & - & - & - \\
\hline 29 & Procyanidin B isomer 3 & $\mathrm{C}_{30} \mathrm{H}_{26} \mathrm{O}_{12}$ & & $57,713,460$ & - & - & - & + & + & - \\
\hline 30 & Digalloylhexose & $\mathrm{C}_{20} \mathrm{H}_{20} \mathrm{O}_{14}$ & & $48,307,749$ & - & - & - & + & + & + \\
\hline $31^{1}$ & $\begin{array}{l}\text { Epigallocatechin-3-O-gallate } \\
\text { (Teatannin II) }\end{array}$ & $\mathrm{C}_{22} \mathrm{H}_{18} \mathrm{O}_{11}$ & & $45,707,709$ & - & + & + & + & + & + \\
\hline $32^{1}$ & Epicatechin & $\mathrm{C}_{15} \mathrm{H}_{14} \mathrm{O}_{6}$ & & $28,907,121$ & - & + & + & + & + & + \\
\hline 33 & Punicacortein $\mathrm{C}$ or $\mathrm{D}$ & $\mathrm{C}_{48} \mathrm{H}_{28} \mathrm{O}_{30}$ & & $108,305,872$ & - & - & - & + & + & + \\
\hline
\end{tabular}


Table 3. Cont

\begin{tabular}{|c|c|c|c|c|c|c|c|c|c|c|}
\hline No. & Name & Formula & {$[\mathrm{M}+\mathrm{H}]^{+}$} & {$[\mathbf{M}-\mathbf{H}]^{-}$} & Leaves-EA & Leaves-MeOH & Leaves-Water & $\begin{array}{c}\text { Stem } \\
\text { Bark-EA }\end{array}$ & $\begin{array}{c}\text { Stem } \\
\text { Bark-MeOH }\end{array}$ & $\begin{array}{c}\text { Stem } \\
\text { Bark-Water }\end{array}$ \\
\hline 34 & Trigalloylhexose isomer 1 & $\mathrm{C}_{27} \mathrm{H}_{24} \mathrm{O}_{18}$ & & $63,508,844$ & - & - & - & + & + & + \\
\hline 35 & Trigalloylhexose isomer 2 & $\mathrm{C}_{27} \mathrm{H}_{24} \mathrm{O}_{18}$ & & $63,508,844$ & - & - & - & + & + & + \\
\hline 36 & Di-O-methylcoruleoellagic acid & $\mathrm{C}_{16} \mathrm{H}_{10} \mathrm{O}_{10}$ & & $36,101,958$ & - & - & - & + & - & - \\
\hline 37 & Mangiferin & $\mathrm{C}_{19} \mathrm{H}_{18} \mathrm{O}_{11}$ & & $42,107,709$ & - & - & - & + & + & + \\
\hline 38 & Trigalloylhexose isomer 3 & $\mathrm{C}_{27} \mathrm{H}_{24} \mathrm{O}_{18}$ & & $63,508,844$ & - & - & - & + & + & + \\
\hline $39^{1}$ & Taxifolin (Dihydroquercetin) & $\mathrm{C}_{15} \mathrm{H}_{12} \mathrm{O}_{7}$ & & $30,305,048$ & + & + & + & + & + & + \\
\hline 40 & Ferulic acid & $\mathrm{C}_{10} \mathrm{H}_{10} \mathrm{O}_{4}$ & & $19,305,009$ & + & + & + & - & - & - \\
\hline 41 & Tetragalloylhexose & $\mathrm{C}_{34} \mathrm{H}_{28} \mathrm{O}_{22}$ & & $78,709,940$ & - & - & - & + & + & - \\
\hline $42^{1}$ & Epicatechin-3-O-gallate & $\mathrm{C}_{22} \mathrm{H}_{18} \mathrm{O}_{10}$ & & $44,108,218$ & + & + & + & + & + & + \\
\hline 43 & Chebulagic acid & $\mathrm{C}_{41} \mathrm{H}_{30} \mathrm{O}_{27}$ & & $95,308,963$ & + & + & + & - & - & - \\
\hline 44 & Ellagic acid O-glucuronide & $\mathrm{C}_{20} \mathrm{H}_{14} \mathrm{O}_{14}$ & & $47,703,054$ & - & - & - & + & + & + \\
\hline 45 & Ellagic acid O-hexoside isomer 1 & $\mathrm{C}_{20} \mathrm{H}_{16} \mathrm{O}_{13}$ & & $46,305,127$ & + & + & + & + & + & + \\
\hline 46 & Ellagic acid O-hexoside isomer 2 & $\mathrm{C}_{20} \mathrm{H}_{16} \mathrm{O}_{13}$ & & $46,305,127$ & + & + & + & + & + & + \\
\hline 47 & Coumaroylshikimic acid & $\mathrm{C}_{16} \mathrm{H}_{16} \mathrm{O}_{7}$ & & $31,908,178$ & + & + & + & - & - & - \\
\hline 48 & $\begin{array}{l}\text { O-Methylellagic acid O-hexoside } \\
\text { isomer } 1\end{array}$ & $\mathrm{C}_{21} \mathrm{H}_{18} \mathrm{O}_{13}$ & & $47,706,692$ & - & - & - & + & + & + \\
\hline 49 & Ellagic acid C-hexoside isomer 1 & $\mathrm{C}_{20} \mathrm{H}_{16} \mathrm{O}_{13}$ & $46,506,692$ & & - & - & - & + & + & + \\
\hline 50 & Myricetin-O-hexoside & $\mathrm{C}_{21} \mathrm{H}_{20} \mathrm{O}_{13}$ & & $47,908,257$ & + & + & + & + & + & + \\
\hline 51 & Quercetin-O-galloylhexoside & $\mathrm{C}_{28} \mathrm{H}_{24} \mathrm{O}_{16}$ & & $61,509,862$ & + & + & + & - & - & - \\
\hline 52 & Vitexin (Apigenin-8-C-glucoside) & $\mathrm{C}_{21} \mathrm{H}_{20} \mathrm{O}_{10}$ & $43,311,348$ & & - & - & - & + & + & + \\
\hline 53 & Pentagalloylhexose & $\mathrm{C}_{41} \mathrm{H}_{32} \mathrm{O}_{26}$ & & $93,911,036$ & - & - & - & + & + & - \\
\hline 54 & $\begin{array}{c}\text { Di-O-methylflavellagic acid } \\
\text { O-hexoside }\end{array}$ & $\mathrm{C}_{22} \mathrm{H}_{20} \mathrm{O}_{14}$ & & $50,707,749$ & - & - & - & + & + & + \\
\hline 55 & Theaflavin or isomer & $\mathrm{C}_{29} \mathrm{H}_{24} \mathrm{O}_{12}$ & $56,513,461$ & & - & + & - & - & + & - \\
\hline 56 & $\begin{array}{l}\text { O-Methylellagic acid O-hexoside } \\
\text { isomer } 2\end{array}$ & $\mathrm{C}_{21} \mathrm{H}_{18} \mathrm{O}_{13}$ & & $47,706,692$ & - & - & - & + & + & + \\
\hline 57 & $\begin{array}{c}\text { Aromadendrin } \\
\text { (Dihydrokaempferol) }\end{array}$ & $\mathrm{C}_{15} \mathrm{H}_{12} \mathrm{O}_{6}$ & & $28,705,557$ & + & + & + & + & + & - \\
\hline 58 & $\begin{array}{l}\text { 3,3'-Di-O-methylellagic } \\
\text { acid-4-O-glucoside }\end{array}$ & $\mathrm{C}_{22} \mathrm{H}_{20} \mathrm{O}_{13}$ & & $49,108,257$ & + & + & + & + & + & + \\
\hline 59 & Quercetin-3-O-glucuronide & $\mathrm{C}_{21} \mathrm{H}_{18} \mathrm{O}_{13}$ & & $47,706,692$ & + & + & + & - & - & - \\
\hline 60 & $\begin{array}{l}\text { Isoquercitrin (Hirsutrin, } \\
\text { Quercetin-3-O-glucoside) }\end{array}$ & $\mathrm{C}_{21} \mathrm{H}_{20} \mathrm{O}_{12}$ & & $46,308,765$ & + & + & + & - & - & - \\
\hline 61 & Rutin (Quercetin-3-O-rutinoside) & $\mathrm{C}_{27} \mathrm{H}_{30} \mathrm{O}_{16}$ & $61,116,122$ & & + & + & + & - & - & - \\
\hline 62 & $\begin{array}{l}\text { Luteolin-7-O-glucoside } \\
\text { (Cynaroside) }\end{array}$ & $\mathrm{C}_{21} \mathrm{H}_{20} \mathrm{O}_{11}$ & & $44,709,274$ & - & - & - & - & - & + \\
\hline
\end{tabular}


Table 3. Cont

\begin{tabular}{|c|c|c|c|c|c|c|c|c|c|c|}
\hline No. & Name & Formula & {$[\mathrm{M}+\mathrm{H}]^{+}$} & {$[\mathbf{M}-\mathbf{H}]^{-}$} & Leaves-EA & Leaves-MeOH & Leaves-Water & $\begin{array}{c}\text { Stem } \\
\text { Bark-EA }\end{array}$ & $\begin{array}{c}\text { Stem } \\
\text { Bark-MeOH }\end{array}$ & $\begin{array}{c}\text { Stem } \\
\text { Bark-Water }\end{array}$ \\
\hline 63 & Luteolin-O-deoxyhexosylhexoside & $\mathrm{C}_{27} \mathrm{H}_{30} \mathrm{O}_{15}$ & & $59,315,065$ & - & - & - & - & - & + \\
\hline 64 & $\begin{array}{c}\text { Isovitexin } \\
\text { (Apigenin-6-C-glucoside) }\end{array}$ & $\mathrm{C}_{21} \mathrm{H}_{20} \mathrm{O}_{10}$ & $43,311,348$ & & - & - & - & + & + & + \\
\hline 65 & Coatline A isomer & $\mathrm{C}_{21} \mathrm{H}_{24} \mathrm{O}_{10}$ & & $43,512,913$ & - & + & + & + & + & - \\
\hline 66 & Ellagic acid O-pentoside & $\mathrm{C}_{19} \mathrm{H}_{14} \mathrm{O}_{12}$ & & $43,304,071$ & - & - & - & + & + & + \\
\hline 67 & Ellagic acid C-hexoside isomer 2 & $\mathrm{C}_{20} \mathrm{H}_{16} \mathrm{O}_{13}$ & $46,506,692$ & & - & - & - & + & + & + \\
\hline 68 & $\begin{array}{l}\text { Eschweilenol C (Ellagic } \\
\text { acid-4-O-rhamnoside) }\end{array}$ & $\mathrm{C}_{20} \mathrm{H}_{16} \mathrm{O}_{12}$ & & $44,705,636$ & + & + & + & + & + & + \\
\hline 69 & Reinutrin (Quercetin-3-O-xyloside) & $\mathrm{C}_{20} \mathrm{H}_{18} \mathrm{O}_{11}$ & & $43,307,709$ & + & + & + & - & - & - \\
\hline 70 & Ellagic acid & $\mathrm{C}_{14} \mathrm{H}_{6} \mathrm{O}_{8}$ & & $30,099,845$ & + & + & + & + & + & + \\
\hline 71 & $\begin{array}{c}\text { Avicularin } \\
\text { (Quercetin-3-O-arabinoside) }\end{array}$ & $\mathrm{C}_{20} \mathrm{H}_{18} \mathrm{O}_{11}$ & & $43,307,709$ & + & + & + & - & - & - \\
\hline $72^{1}$ & $\begin{array}{c}\text { Myricetin } \\
\left(3,3^{\prime}, 4^{\prime}, 5,5^{\prime}, 7-\text { Hexahydroxyflavone }\right)\end{array}$ & $\mathrm{C}_{15} \mathrm{H}_{10} \mathrm{O}_{8}$ & & $31,702,974$ & + & + & + & + & + & + \\
\hline 73 & $\begin{array}{c}\text { Guaijaverin } \\
\text { (Quercetin-3-O-arabinoside) }\end{array}$ & $\mathrm{C}_{20} \mathrm{H}_{18} \mathrm{O}_{11}$ & & $43,307,709$ & + & + & + & - & - & - \\
\hline 74 & $\begin{array}{l}\text { Isorhamnetin-O-glucuronide } \\
\text { isomer } 1\end{array}$ & $\mathrm{C}_{22} \mathrm{H}_{20} \mathrm{O}_{13}$ & & $49,108,257$ & - & + & - & - & - & - \\
\hline 75 & $\begin{array}{c}\text { Quercitrin } \\
\text { (Quercetin-3-O-rhamnoside) }\end{array}$ & $\mathrm{C}_{21} \mathrm{H}_{20} \mathrm{O}_{11}$ & & $44,709,274$ & + & + & + & - & - & - \\
\hline 76 & $\begin{array}{l}\text { Di-O-methylflavellagic acid } \\
\text { O-pentoside }\end{array}$ & $\mathrm{C}_{21} \mathrm{H}_{18} \mathrm{O}_{13}$ & & $47,706,692$ & - & - & - & + & - & - \\
\hline 77 & $\begin{array}{c}\text { Ducheside A (3-O-Methylellagic } \\
\text { acid-4'-O-xyloside) }\end{array}$ & $\mathrm{C}_{20} \mathrm{H}_{16} \mathrm{O}_{12}$ & & $44,705,636$ & - & - & - & + & + & + \\
\hline 78 & Eriodictyol & $\mathrm{C}_{15} \mathrm{H}_{12} \mathrm{O}_{6}$ & & $28,705,557$ & + & + & - & + & + & + \\
\hline 79 & Isorhamnetin-3-O-glucoside & $\mathrm{C}_{22} \mathrm{H}_{22} \mathrm{O}_{12}$ & & $47,710,330$ & + & + & + & - & - & - \\
\hline 80 & $\begin{array}{l}\text { Dimethoxy-tetrahydroxy(iso) } \\
\text { flavone-O-hexoside }\end{array}$ & $\mathrm{C}_{23} \mathrm{H}_{24} \mathrm{O}_{13}$ & & $50,711,387$ & - & + & + & - & - & - \\
\hline 81 & $\begin{array}{l}\text { Isorhamnetin-O-glucuronide } \\
\text { isomer } 2\end{array}$ & $\mathrm{C}_{22} \mathrm{H}_{20} \mathrm{O}_{13}$ & & $49,108,257$ & - & + & - & - & - & - \\
\hline 82 & Isorhamnetin-O-glucuronide & $\mathrm{C}_{22} \mathrm{H}_{20} \mathrm{O}_{13}$ & & $49,108,257$ & - & - & + & - & - & - \\
\hline 83 & $\begin{array}{l}\text { Isorhamnetin-3-O-rutinoside } \\
\text { (Narcissin) }\end{array}$ & $\mathrm{C}_{28} \mathrm{H}_{32} \mathrm{O}_{16}$ & & $62,316,122$ & + & + & + & - & - & - \\
\hline 84 & 4-Methoxycinnamic acid & $\mathrm{C}_{10} \mathrm{H}_{10} \mathrm{O}_{3}$ & & $17,907,082$ & - & - & - & - & + & - \\
\hline 85 & $\begin{array}{l}\text { Di-O-methylellagic } \\
\text { acid-O-pentoside }\end{array}$ & $\mathrm{C}_{21} \mathrm{H}_{18} \mathrm{O}_{12}$ & & $46,107,200$ & + & + & + & + & + & + \\
\hline
\end{tabular}


Table 3. Cont

\begin{tabular}{|c|c|c|c|c|c|c|c|c|c|c|}
\hline No. & Name & Formula & {$[\mathrm{M}+\mathrm{H}]^{+}$} & {$[\mathbf{M}-\mathbf{H}]^{-}$} & Leaves-EA & Leaves-MeOH & Leaves-Water & $\begin{array}{c}\text { Stem } \\
\text { Bark-EA }\end{array}$ & $\begin{array}{c}\text { Stem } \\
\text { Bark-MeOH }\end{array}$ & $\begin{array}{c}\text { Stem } \\
\text { Bark-Water }\end{array}$ \\
\hline 86 & $\begin{array}{l}\text { 3,3',4-Tri-O-methylflavellagic } \\
\text { acid-4-O-glucoside }\end{array}$ & $\mathrm{C}_{23} \mathrm{H}_{22} \mathrm{O}_{14}$ & & $52,109,314$ & + & + & + & + & + & + \\
\hline 87 & Quercetin & $\mathrm{C}_{15} \mathrm{H}_{10} \mathrm{O}_{7}$ & & $30,103,483$ & + & + & + & - & - & - \\
\hline 88 & 3-O-Methylellagic acid & $\mathrm{C}_{15} \mathrm{H}_{8} \mathrm{O}_{8}$ & & $31,501,410$ & - & + & + & + & + & + \\
\hline 89 & $\begin{array}{l}\text { Di-O-methylellagic } \\
\text { acid-O-deoxyhexoside }\end{array}$ & $\mathrm{C}_{22} \mathrm{H}_{20} \mathrm{O}_{12}$ & & $47,508,766$ & - & - & - & + & + & + \\
\hline $90^{1}$ & Naringenin & $\mathrm{C}_{15} \mathrm{H}_{12} \mathrm{O}_{5}$ & & $27,106,065$ & + & + & + & + & + & + \\
\hline 91 & $\begin{array}{l}\text { Di-O-methylflavellagic acid isomer } \\
1\end{array}$ & $\mathrm{C}_{16} \mathrm{H}_{10} \mathrm{O}_{9}$ & & $34,502,466$ & - & - & - & + & + & + \\
\hline $92^{1}$ & $\begin{array}{c}\text { Luteolin } \\
\left(3^{\prime}, 4^{\prime}, 5,7-\text { Tetrahydroxyflavone }\right)\end{array}$ & $\mathrm{C}_{15} \mathrm{H}_{10} \mathrm{O}_{6}$ & & $28,503,991$ & + & + & + & - & + & - \\
\hline 93 & 3,3'-Di-O-methylellagic acid & $\mathrm{C}_{16} \mathrm{H}_{10} \mathrm{O}_{8}$ & & $32,902,975$ & + & + & + & + & + & + \\
\hline 94 & $\begin{array}{c}\text { Kaempferol } \\
\left(3,4^{\prime}, 5,7-\text { Tetrahydroxyflavone }\right)\end{array}$ & $\mathrm{C}_{15} \mathrm{H}_{10} \mathrm{O}_{6}$ & $28,705,556$ & & + & + & + & - & - & - \\
\hline 95 & $\begin{array}{c}\text { Isorhamnetin ( } 3^{\prime} \text {-Methoxy-3,4', } 5,7 \\
\text {-tetrahydroxyflavone) }\end{array}$ & $\mathrm{C}_{16} \mathrm{H}_{12} \mathrm{O}_{7}$ & & $31,505,048$ & + & + & + & - & - & - \\
\hline 96 & Dimethoxy-trihydroxy(iso)flavone & $\mathrm{C}_{17} \mathrm{H}_{14} \mathrm{O}_{7}$ & & $32,906,613$ & + & + & - & - & - & - \\
\hline 97 & $\begin{array}{l}\text { Di-O-methylflavellagic acid isomer } \\
2\end{array}$ & $\mathrm{C}_{16} \mathrm{H}_{10} \mathrm{O}_{9}$ & & $34,502,466$ & - & - & - & + & + & + \\
\hline 98 & $\begin{array}{c}\text { Tetra-O-methylflavellagic acid } \\
\text { isomer } 1\end{array}$ & $\mathrm{C}_{18} \mathrm{H}_{14} \mathrm{O}_{9}$ & & $37,305,596$ & - & - & - & + & + & + \\
\hline 99 & $\begin{array}{c}\text { Apigenin } \\
\left(4^{\prime}, 5,7-\text { Trihydroxyflavone }\right)\end{array}$ & $\mathrm{C}_{15} \mathrm{H}_{10} \mathrm{O}_{5}$ & & $26,904,500$ & - & + & - & - & - & + \\
\hline 100 & $3,3^{\prime}, 4$-Tri-O-methylellagic acid & $\mathrm{C}_{17} \mathrm{H}_{12} \mathrm{O}_{8}$ & & $34,304,540$ & + & + & + & + & + & + \\
\hline 101 & Undecanedioic acid & $\mathrm{C}_{11} \mathrm{H}_{20} \mathrm{O}_{4}$ & & $21,512,834$ & + & + & + & - & - & - \\
\hline 102 & $\begin{array}{c}\text { Tetra-O-methylflavellagic acid } \\
\text { isomer } 2\end{array}$ & $\mathrm{C}_{18} \mathrm{H}_{14} \mathrm{O}_{9}$ & & $37,305,596$ & - & - & - & + & + & + \\
\hline 103 & 3,3',4-Tri-O-methylflavellagic acid & $\mathrm{C}_{17} \mathrm{H}_{12} \mathrm{O}_{9}$ & & $35,904,031$ & + & + & + & + & + & + \\
\hline 104 & Dihydroxy-dimethoxy(iso)flavone & $\mathrm{C}_{17} \mathrm{H}_{14} \mathrm{O}_{6}$ & & $31,307,122$ & + & + & - & - & - & - \\
\hline 105 & $\begin{array}{c}\text { Pinocembrin } \\
\text { (5,7-Dihydroxyflavanone) }\end{array}$ & $\mathrm{C}_{15} \mathrm{H}_{12} \mathrm{O}_{4}$ & & $25,506,573$ & + & + & - & - & + & - \\
\hline 106 & Dihydroxy-trimethoxy(iso)flavone & $\mathrm{C}_{18} \mathrm{H}_{16} \mathrm{O}_{7}$ & & $34,308,178$ & + & + & + & - & - & - \\
\hline 107 & Dodecanedioic acid & $\mathrm{C}_{12} \mathrm{H}_{22} \mathrm{O}_{4}$ & & $22,914,399$ & + & + & + & - & - & - \\
\hline 108 & Hexadecanedioic acid & $\mathrm{C}_{16} \mathrm{H}_{30} \mathrm{O}_{4}$ & & $28,520,659$ & + & + & - & - & - & - \\
\hline
\end{tabular}

${ }^{1}$ Confirmed by standard. 


\subsection{Phenolic Acids}

Quinic $\left([\mathrm{M}-\mathrm{H}]^{-}\right.$at $\left.m / z 191\right)$, shikimic $\left([\mathrm{M}-\mathrm{H}]^{-}\right.$at $\left.m / z 173\right)$, gallic $\left([\mathrm{M}-\mathrm{H}]^{-}\right.$at $\left.m / z 169\right)$, and protocatechuic $\left([\mathrm{M}-\mathrm{H}]^{-}\right.$at $\left.m / z 153\right)$ acids were tentatively identified from all the extracts of $A$. leiocarpus leaves and stem bark. Chlorogenic $\left([\mathrm{M}-\mathrm{H}]^{+}\right.$at $\left.m / z 355\right)$, caffeic $\left([\mathrm{M}-\mathrm{H}]^{-}\right.$at $\left.m / z 179\right)$, ferulic $\left([\mathrm{M}-\mathrm{H}]^{-}\right.$ at $m / z$ 193), and dodecanedioic $\left([\mathrm{M}-\mathrm{H}]^{-}\right.$at $\left.m / z 229\right)$ acids were tentatively identified from the leaves extracts of A. leiocarpus only.

\subsection{Flavonoids}

Several compounds belonging to the flavonoid family were identified from A. leiocarpus extracts. The compound suffering deprotonation at $m / z 269[\mathrm{M}-\mathrm{H}]^{-}$and fragment ions at $m / z 225,151,149$, and 117 was characterised as apigenin and was present in the methanol extract of $A$. leiocarpus leaves and water extract of $A$. leiocarpus stem bark. C-glucosides of apigenin $\left([\mathrm{M}-\mathrm{H}]^{+}\right.$at $\left.m / z 433\right)$, namely, vitexin and isovitexin, only occurred in the stem bark extracts of $A$. leiocarpus. Luteolin, suffering deprotonation at $m / z 285[\mathrm{M}-\mathrm{H}]^{-}$and fragment ions at $m / z 217,199,175,151$, and 133 was tentatively characterised. Other flavonoids, such as, catechin $\left([\mathrm{M}-\mathrm{H}]^{-}\right.$at $\left.m / z 289\right)$, naringenin $\left([\mathrm{M}-\mathrm{H}]^{-}\right.$at $m / z 271)$, myricetin $\left([\mathrm{M}-\mathrm{H}]^{-}\right.$at $\left.m / z 317\right)$, taxifolin $\left([\mathrm{M}-\mathrm{H}]^{-}\right.$at $\left.m / z 303\right)$, and pinocembrin $\left([\mathrm{M}-\mathrm{H}]^{-}\right.$at $m / z 255)$ were also identified.

\subsection{Tannins Derivatives}

Some tannin derivatives were tentatively characterised. Casuarinin with deprotonation at $\mathrm{m} / \mathrm{z}$ $935[\mathrm{M}-\mathrm{H}]^{-}$and fragment ions at $m / z 917,783,633,300$, and 275, was tentatively characterized in all the extracts. Hydrolysable tannins, namely chebulagic acid and punicalagin [27], were characterized at $[\mathrm{M}-\mathrm{H}]^{-} \mathrm{m} / \mathrm{z} 953$ and 1083, respectively. While punicalagin was identified in all of the extracts, chebulagic acid was only characterised in the leaves extracts.

\subsection{Antioxidant Activities}

Phytochemicals, which are ubiquitously present in plants, have been identified to possess antioxidant activity and they are capable of managing oxidative stress related diseases [28]. In this study, three types of antioxidant mechanisms were used, namely, radical scavenging, reducing power, and metal chelating. Table 4 presents the ability of $A$. leiocarpus extracts to scavenge DPPH and ABTS radicals. DPPH, which is a stable radical, is widely used to assess the free radical scavenging abilities of plant extract. By proton transfer, there is the DPPH change in the non-radical form, characterized by a yellow chromophore [29]. On the other side, the ABTS method is based on the monitoring of electron or hydrogen transfer-induced ABTS radical-cation decay, which is characterized by the disappearance of the corresponding blue-green radical [30]. From Table 4, it is observed that, in general, the methanol and water extracts of $A$. leiocarpus leaves and stem bark showed potent radical scavenging properties compared to the ethyl acetate extracts of A. leiocarpus leaves and stem bark. Besides, it was noted that the stem bark extracts were more potent radical scavengers when compared to the leaves extracts. Furthermore, the strong radical scavenging activities of the extracts was related to their high phenolic contents. Reducing power, as described by electron transfer ability, is considered to be one of the key indicators of antioxidant capacity of plant extracts [28]. In this study, the reducing power of A. leiocarpus extracts was assessed using FRAP and CUPRAC assays, which are characterized by the reduction of $\mathrm{Fe}^{3+}$ to $\mathrm{Fe}^{2+}$ and $\mathrm{Cu}^{2+}$ to $\mathrm{Cu}^{+}$, respectively $[30,31]$. Comparable to the free radical scavenging assessment, the methanol and water extracts of A. leiocarpus showed potent reducing properties as compared to the ethyl acetate extracts. The total antioxidant capacity that was determined by the phosphomolybdenum assay demonstrated that methanol and water extracts of $A$. leiocarpus leaves and stem bark were more potent antioxidants as compared to the ethyl acetate extracts. This finding is in line with radical scavenging and reducing power evaluations. The ability of $A$. leiocarpus extracts to chelate metal was also evaluated and is presented in Table 4. Given the recognised role of iron in 
oxidative stress, which is understood as an increase in oxygen radical intermediates concentration, eventually leading to dysregulation, the development of metal chelators having the ability to restore metal homeostasis and oxidative status appears to be a valuable challenge, particularly if the chelators possess other important biological activities that might mitigate other diseases [32]. Data that were gathered from this study revealed that the water extract of $A$. leiocarpus leaves, possessing the highest flavonoid content, was the most activity metal chelator. It has previously been proposed that flavonoids were potent chelators of iron [33]. Naringenin, quercetin, luteolin, and catechin compounds, belonging to the flavonoid family, were identified in the water extract of A. leiocarpus leaves and they were reported to possess metal chelating abilities [33,34]. For instance, quercetin was reported to form different complexes with $\mathrm{Fe}^{2+}$ through its 5-OH and 4-carbonyl groups [35].

Table 4. Antioxidant properties of A. leiocarpus extracts.

\begin{tabular}{|c|c|c|c|c|c|c|}
\hline Samples & $\begin{array}{l}\text { DPPH } \\
\text { (mmol TE/g) }\end{array}$ & $\begin{array}{l}\text { ABTS } \\
(\mathrm{mmol} \mathrm{TE} / \mathrm{g})\end{array}$ & $\begin{array}{l}\text { CUPRAC } \\
(\mathrm{mmol} \mathrm{TE} / \mathrm{g})\end{array}$ & $\begin{array}{l}\text { FRAP } \\
\text { (mmol TE/g) }\end{array}$ & $\begin{array}{l}\text { Metal Chelating } \\
\text { (mg EDTAE/g) }\end{array}$ & $\begin{array}{l}\text { Phosphomolybdenum } \\
\text { (mmol TE/g) }\end{array}$ \\
\hline Leaves-EA & $30 \pm 0.01^{f}$ & $0.26 \pm 0.02^{\mathrm{e}}$ & $0.50 \pm 0.04^{\mathrm{e}}$ & $0.26 \pm 0.03^{\mathrm{d}}$ & $10.0 \pm 0.8^{f}$ & $2.0 \pm 0.1^{\mathrm{e}}$ \\
\hline Leaves-MeOH & $5.0 \pm 0.1^{\mathrm{d}}$ & $3.0 \pm 0.4^{c}$ & $7.0 \pm 0.2^{c}$ & $4.0 \pm 0.1^{\mathrm{c}}$ & $47.0 \pm 0.8^{c}$ & $4.0 \pm 0.1^{\mathrm{d}}$ \\
\hline Leaves-Water & $5.0 \pm 0.1^{\mathrm{c}}$ & $4.0 \pm 0.3^{\mathrm{b}}$ & $7.0 \pm 0.2^{b}$ & $6.0 \pm 0.4^{\mathrm{b}}$ & $79.0 \pm 0.9^{a}$ & $4.0 \pm 0.1^{\mathrm{d}}$ \\
\hline Stem barks-EA & $3.0 \pm 0.1^{\mathrm{e}}$ & $2.0 \pm 0.1^{\mathrm{d}}$ & $5.0 \pm 0.2^{\mathrm{d}}$ & $4.0 \pm 0.1^{\mathrm{c}}$ & $30.0 \pm 0.5^{\mathrm{e}}$ & $4.0 \pm 0.1^{\mathrm{c}}$ \\
\hline Stem barks-MeOH & $6.0 \pm 0.1^{\mathrm{a}}$ & $5.0 \pm 0.1^{\mathrm{a}}$ & $8.0 \pm 0.2^{\mathrm{a}}$ & $6.0 \pm 0.3^{\mathrm{a}}$ & $45.0 \pm 0.6^{\mathrm{d}}$ & $6.0 \pm 0.2^{\mathrm{a}}$ \\
\hline Stem barks-Water & $5.0 \pm 0.1^{\mathrm{b}}$ & $4.0 \pm 0.4^{\mathrm{b}}$ & $8.0 \pm 0.1^{b}$ & $6.0 \pm 0.2^{\mathrm{a}}$ & $61.0 \pm 0.4^{b}$ & $5.0 \pm 0.1^{\mathrm{a}}$ \\
\hline
\end{tabular}

Values expressed are means \pm S.D. of three parallel measurements. DPPH: 2-diphenyl-1-picrylhydrazyl; ABTS: 2,2'-azino-bis(3-ethylbenzothiazoline-6-sulphonic acid; CUPRAC: cupric reducing antioxidant capacity; FRAP: ferric reducing antioxidant power; TE: Trolox equivalent; EDTAE: ethylenediaminetetraacetic acid equivalent. Superscripts in the same column indicate significant difference in the tested extracts $(p<0.05)$.

\subsection{Enzyme Inhibitory Activities}

While the antioxidant activity of plant extract is often linked to the phenolic content, the enzyme inhibitory properties of extracts mainly involves the interaction of phytochemicals with the enzyme or enzyme-substrate complex. To the best of our knowledge, this is the first report on the assessment of the inhibitory potential of A. leiocarpus leaves and stem bark on enzymes related to Alzheimer's disease and skin hyperpigmentation. A previous study has appraised the amylase and glucosidase inhibitory action of $A$. leiocarpus leaves [5]. However, no comparison has been made with the stem bark extract of the plant and the possible effect of different extraction solvents. Among the five food drug administration (FDA)-approved Alzheimer's disease treatments, four are acetyl cholinesterase inhibitors [36]. Cholinesterase inhibitors designed for the management of Alzheimer's disease stem from the cholinergic hypothesis, which is the leading theory proposed to explain the pathogenesis of Alzheimer's disease [37]. It has been recognised that cholinergic neurons loss in brain area that is responsible for cognition and behaviour was the hallmark of Alzheimer's disease. While the role of acetyl cholinesterase has been clearly claimed, the exact mechanism that involves butyryl cholinesterase remains elusive. Butyryl cholinesterase, previously underestimated in the pathogenesis of Alzheimer's disease, was found to be up-regulated in advanced stages of the condition and plays a key role in the disease maintenance and progression [38]. From this perspective, it can be stated that cholinesterase inhibitors targeting both acetyl and butyryl cholinesterases are in need. Table 5 reports the acetyl cholinesterase inhibitory activity of the different extracts of $A$. leiocarpus leaves and stem bark ranging from 3.51 to $4.68 \mathrm{mg}$ GALAE/g. With regards to the butyryl cholinesterase inhibitory action, the values ranged from 0.45 to $4.0 \mathrm{mg}$ GALAE/g. Interestingly, the water extract of A. leiocarpus leaves only inhibited acetyl cholinesterase. It can be suggested that this extract might be targeted at the initial stage of the disease, when butyryl cholinesterase activity is not pronounced. The methanol extract of A. leiocarpus leaves (4.68 and $4.0 \mathrm{mg}$ GALAE/g) showed potent inhibition against both cholinesterases. Over the past decades, there has been an emerging trend of naturally derived cosmetic products. This shift has encouraged researchers to find new cosmeceuticals and the focus has geared towards plants. Plant extracts have witnessed increased global demand for de-pigmenting agents due to their safety and compatibility with all skin types [39]. The inhibitory action of $A$. leiocarpus extracts on tyrosinase, a 
copper-containing enzyme responsible for the biosynthesis of melanin [40], was investigated. The data collected showed potent tyrosinase inhibition with values ranging from 113.0 to $155.26 \mathrm{mg} \mathrm{KAE} / \mathrm{g}$, the highest values was recorded for methanol extract of $A$. leiocarpus stem bark. Pinocembrin, shikimic acid, and vitexin, tentatively identified in the methanol extract of $A$. leiocarpus stem bark, were previously reported to inhibit tyrosinase [41-43]. A group of researchers [5] have reported the amylase $\left(\mathrm{IC}_{50}\right.$ value of $242.17 \mu \mathrm{g} / \mathrm{mL}$ ) and glucosidase ( $\mathrm{IC}_{50}$ value of $196.35 \mu \mathrm{g} / \mathrm{mL}$ ) inhibitory activity of $A$. leiocarpus leaves water extract. In the present investigation and, as opposed to the previous study, low inhibition was recorded against amylase, while no inhibitory action was observed against glucosidase in the presence of the water extract of $A$. leiocarpus leaves. The different activity that was recorded in our study might be related to the geographical location along with the environmental conditions of the studied A. leiocarpus plants. In this study, it was observed that the different A. leiocarpus extracts were poor inhibitors of amylase, with values that ranged from 0.19 to $1.13 \mathrm{mmol} \mathrm{ACAE} / \mathrm{g}$. Only ethyl acetate extracts inhibited glucosidase and the values were higher as compared to amylase. The inhibition of glucosidase is considered as strategic in the management of diabetes type II. Indeed, it has been advocated that the inhibition of glucosidase reduced post-prandial glucose rise and it was associated to less side effects.

Table 5. Enzyme inhibitory properties of A. leiocarpus extracts.

\begin{tabular}{|c|c|c|c|c|c|}
\hline Samples & $\begin{array}{l}\text { AChE Inhibition } \\
\text { (mg GALAE/g) }\end{array}$ & $\begin{array}{l}\text { BChE } \\
\text { Inhibition } \\
\text { (mg GALAE/g) }\end{array}$ & $\begin{array}{l}\text { Tyrosinase } \\
\text { Inhibition } \\
\text { (mg KAE/g) }\end{array}$ & $\begin{array}{l}\text { Amylase } \\
\text { Inhibition } \\
\text { (mmol ACAE/g) }\end{array}$ & $\begin{array}{l}\text { Glucosidase } \\
\text { Inhibition } \\
\text { (mmol ACAE/g) }\end{array}$ \\
\hline Leaves-EA & $4.0 \pm 0.2^{\mathrm{d}}$ & $3.0 \pm 0.2^{b}$ & $131.0 \pm 0.2^{\mathrm{d}}$ & $0.79 \pm 0.04^{\mathrm{b}}$ & $15.0 \pm 0.1^{\mathrm{b}}$ \\
\hline Leaves-MeOH & $4.68 \pm 0.02^{\mathrm{a}}$ & $4.0 \pm 0.1^{\mathrm{a}}$ & $154.0 \pm 0.2^{\mathrm{b}}$ & $1.0 \pm 0.1^{\mathrm{a}}$ & nd \\
\hline Leaves-Water & $4.19 \pm 0.04^{c}$ & nd & $113 \pm 1^{\mathrm{e}}$ & $0.53 \pm 0.04^{c}$ & nd \\
\hline Stem barks-EA & $4.0 \pm 0.1^{\mathrm{bc}}$ & $2.0 \pm 0.2^{c}$ & $152.0 \pm 0.6^{c}$ & $1.0 \pm 0.1^{\mathrm{b}}$ & $15.0 \pm 0.1^{\mathrm{a}}$ \\
\hline Stem barks-MeOH & $4.0 \pm 0.1^{\mathrm{b}}$ & $1.0 \pm 0.3^{c}$ & $155.26 \pm 0.04^{\mathrm{a}}$ & $0.85 \pm 0.03^{b}$ & nd \\
\hline Stem barks-Water & $4.0 \pm 0.1^{\mathrm{e}}$ & $0.5 \pm 0.1^{d}$ & $113.0 \pm 0.6^{\mathrm{e}}$ & $0.19 \pm 0.01^{\mathrm{d}}$ & nd \\
\hline
\end{tabular}

Values expressed are means \pm S.D. of three parallel measurements. AChE: Acetylcholinesterase; BChE: Butyrylcholinesterase; GALAE: Galatamine equivalent; KAE: Kojic acid equivalent; ACAE: Acarbose equivalent. nd: not detected. Superscripts in the same column indicate significant difference in the tested extracts $(p<0.05)$.

\subsection{Multivariate Analysis}

An unsupervised multiple correspondence analysis (MCA), a Heatmap clustering approach, and Venn graph were applied on the chemical composition of A. leiocarpus samples to obtain a typology of the samples and to characterize the chemical profile differences among those samples. MCA is commonly used qualitative variables to examine a set of observations described by a set of nominal variables. Figure 1A displays the proportion of explained inertia per component and the projection of the samples and chemical compounds on the first two dimensions. Two components were required to summarize approximately $97.7 \%$ of the variance. The first component explained $96,1 \%$ of variance, while the second accounted for 1,6\%. As we could notice, the samples were predominantly separated by the first component of MCA, with the extracts of stem bark being grouped on the negative side of the factors and the leaves extract on the positive side (Figure 1B). In agreement with MCA, Heatmap separated and categorized the samples into two groups, with each group being divided into two sub-clusters (Figure 1C). On the other hand, we observed that the stem bark extracts were more homogeneous than the leaves extracts. Indeed, ethyl acetate extract of leaves was clearly separated from the other two extracts (methanol and water), which were close (Figure 1B). This indicates that solvent used have a large influence on the leaves secondary metabolite extraction than that of stem bark. Moreover, when cross-checking a list of 108 compounds that were identified in all the leaves extracts against those were recognized in all stem bark extracts, we observed that a total of 38 were commonly detected in the two organs, whereas there were 38 and 32 unique compounds found in stem bark and leaves, respectively (Figure 2). 


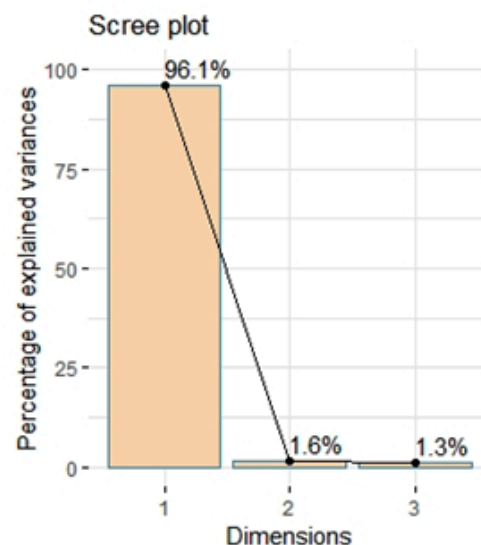

A

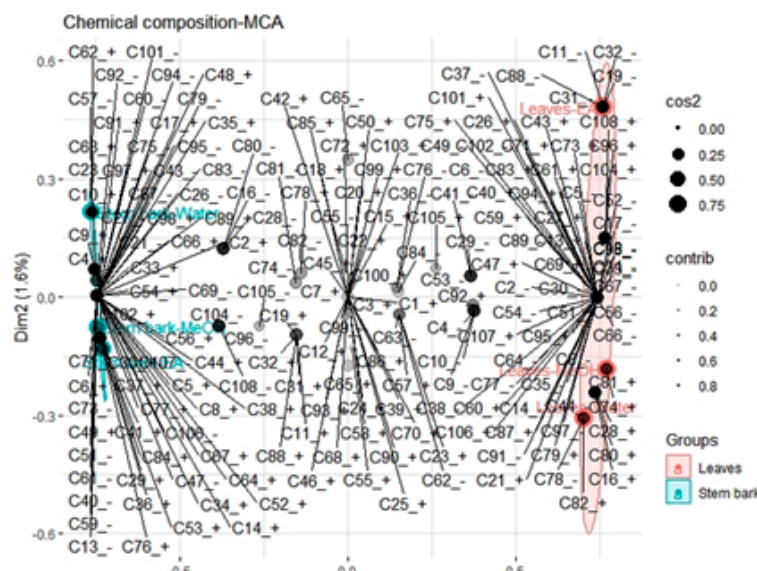

B

$\operatorname{Dim1} 196.1 \%)$
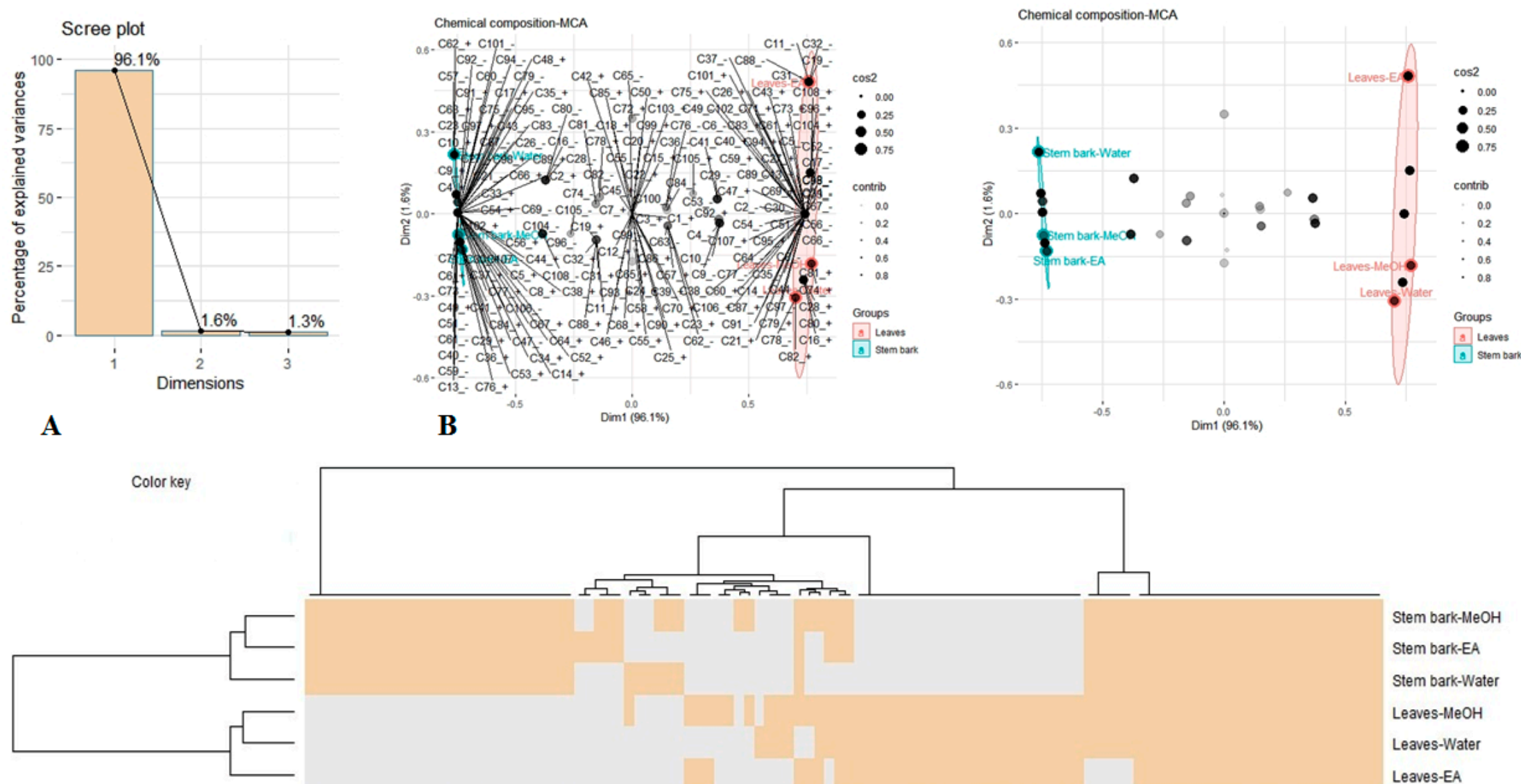

C

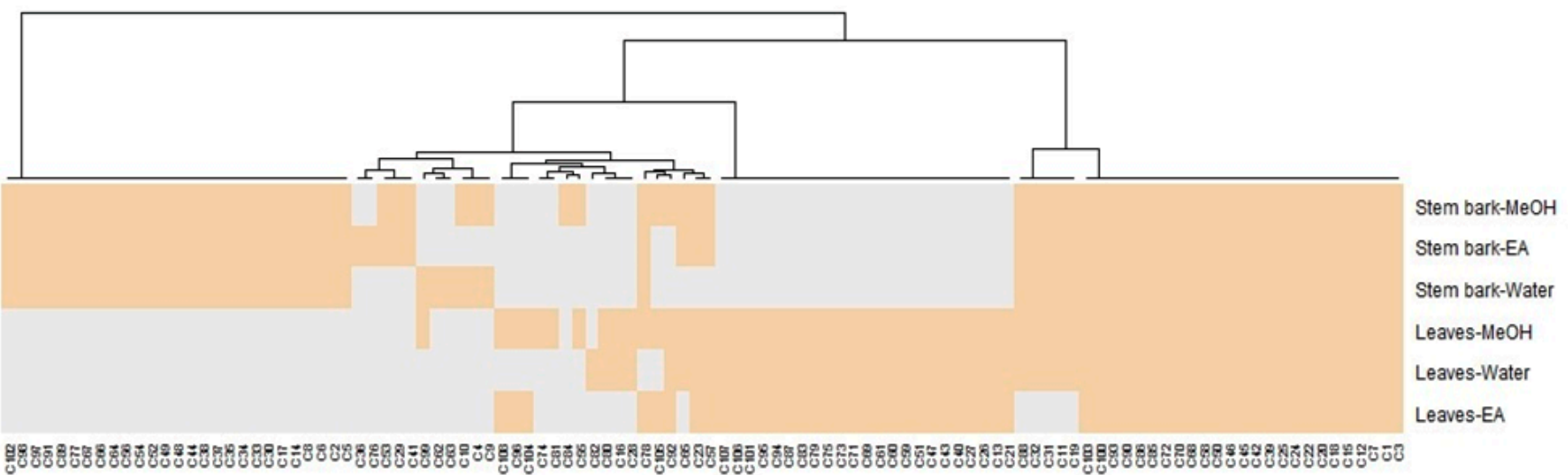

Figure 1. Multivariate analysis using multiple correspondence analysis (MCA) and Heatmap clustering analysis of chemical composition in A. leiocarpus extracts. (A): Percentage of explained variance per component. (B): projection of extracts and chemical compounds into the subspace spanned by the first two components of MCA. (C): Clustered Image Map (Euclidean Distance, Ward linkage). Gray colour: Absence, Wheat colour: Presence. 

C1 Quinic acid
C28 Caffeoylshikimic acid
C55 Theaflavin or isomer
C82 Isorhamnetin-0-giucuronide
C2 Hexahydroxydiphenoyherose C29 Procyanidin B isomer 3
C56 0-Methylellagic acid 0-heroside isomer 2
C83 Isorhamnetin-3-0-rutinoside
C31 Epigallocatechin-3-0-gallate
C58 3,3'-Di-0-methylelagic acid-4-0-glucoside
ethoxycinnamic acid
Galloylquinic acid isomer $1 \quad$ C31 Epigallocatechin-3-0-8d
Galloyherose isomer 1
C32 Epicatechin
C59 Quercetin-3-0-giucuronide
Galloylherose isomer
Gallic acid
Galloyhherose
C33 Punicacortein C or D
C34 Trigalloyherose isomer
C34 Trigalloyherose isomer 1
C35 Trigaloyherose isomer 2
C36 Di-0.-methylcorvileoellagic
C9 Calloyhyuguinic acid isomer 2
C10 Galloylquinic acid isomer 3 C37 Mangiferin
C11 Gallocatechin
C37 Mangiferin
C38 Trigalloyherose isomer
C39 Taxifolin
C60 Isoquercitr
C62 Luteolin-7-0-giucoside
C63 Luteolin-0-deoxyherosylhexoside
C64 Isoviterin
C65 Coattine A is
C65 Coattine A isomer
3 3-Hydroxybenzaldehyde
C40 Ferulic acid
C66 Ellagic acid 0-pentoside
C67 Ellagic acid C-heroside isomer
C68 Eschwelenol C
C69 Reinutrin
C70 Ellagic acid
Tunicalagin
C42 Epicatechin-3-0-gallate
C71 Avicularin
C44 Ellagic acid O-gunuronide
C72 Myricetin
C18 Catechin
C45 Ellagic acid 0-heroside isomer 1
C73 Guajijuerin
C46 Ellagic acid 0-heroside isor
C74 Isorhamnetin-0-gucuronide isomer 1
C86 3,3; -4Tri-0-methylfavellagic acid-40-guncoside
C87 Quercetin 3 3-0-Methylellagic acid
C88 3-0-Methylelalagic acid
C89 Di-0-methylalagic acid-0-deoxyheroside
C90 Naringenin
C91 Di-0-methylfavelagic acid isomer
C92 Luteolin
C93 3,3'-Di-0-methylellagic acid
C94 Kaempferol
C96 Dimethoxy-trihydroxy(iso)flavone
$C 97$ Di-0-methyifavelagic acid isomer 2
C98 Tetra-0-methylfiavelagic acid isomer
C99 Apigenin
C19 Epigallocated
C48 0-Methylelagic acid 0-heroside isomer 1
C75 Quercitrin
C21 Chlorogenic acid
C22 Caffeic acid
C49 Ellagic acid C-heroside isomer 1
C76 Di-0-methyiflavellagic acid 0-pentoside
C100 3,3,4-4ri-0-methylelagic acid
C101 Undecanedioic acid
C102 Tetra-0-methylfivellagic acid isomer 2
C23 Cornusiin B or isomer
C50 Myricetin-0-heroside
C77 Ducheside A
C103 3,3,4-Tri-0-methylfavellagic acid
C24 Ampelopsin
C51 Quercetin-0-galloyheroside
C78 Eriodictyol
C104 Dihydroxy-dimethoxy([iso)fiavone
C25 Tellimagrandin Ior isomer C52 Viterin
C26 Coumaroylquinic acid
C53 Pentagalloyherose
C79 Isorhamnetin-3-0-gucoside
C105 Pinocembrin
C80 Dimethoxy-tetrahydroxy(iso)fiavone-0-heroside C107 Dodecanedioic acid
C27 Corilagin or isomer
C54 Di-0-methylfiavelagic acid 0-heroside
C81 Isorhamnetin-0.gucuronide isomer 2

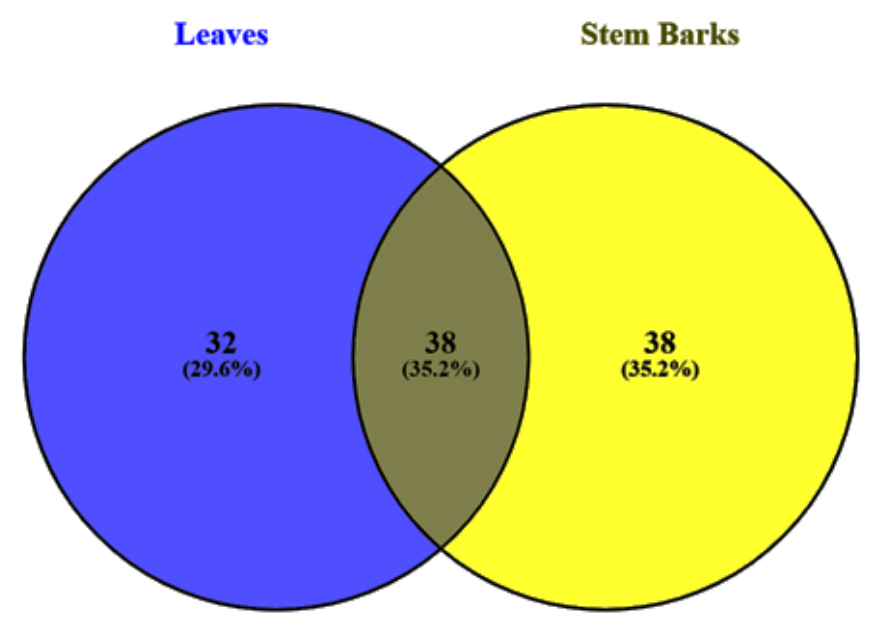

Figure 2. Venn diagram representing the overlap of compounds on the two organs. 
The analysis showed the variation of chemical composition of $A$. leiocarpus depending to part used, as well as the influence of solvent on the extraction of compounds, with a more pronounced effect on the leaves. In agreement with this observation, we decided to ascertain whether the plant parts and solvent types used had any statistically significant effect on both total bioactive compounds content and biological activities of $A$. leiocarpus. Thus, multivariate methods for integrative large biological data sets, namely DIABLO, was applied to total bioactive compounds content and biological activities data-sets. DIABLO is a highly flexible supervised multivariate method that enables to classify in an optimal and reliable manner the studied samples and to construct a predictive multi-omics model that can be used to classify new samples. Figure 3 shows the multivariate analysis results. From Figure $3 \mathrm{~A}, \mathrm{~B}$, it is clear that there were parts and solvents effects on the total bioactive compounds content and biological activities of $A$. leiocarpus. In fact, as we could observe in Figure $3 \mathrm{~A}$, the factorial plan discriminated leaves parts with the stem bark parts effectively in both bioactive compounds and biological activities data sets. A similar outcome was provided with the second studied factor, in short, a clear segregation between the solvents was achieved (Figure 3B). Furthermore, a better separation of solvents was found while using biological activities data than when using the bioactive compounds data. The different extracts of the stem bark were relatively close as we have seen on the plots. As well by observing the samples plot using bioactive compounds content data (Figure 3A,B, Block: Bioactive compounds), this view was echoed with a consolidation of stem bark extracts contrast to a high variability between the leaves extracts. Accordingly, the extraction solvent, by extension the change of polarity, greatly influenced the bioactive compounds content of leaves than those of stem bark. The present result indicated that $A$. leiocarpus leaves, unlike stem bark, contain chemical molecules with varying polarity and solubility that are sensitive to the variation of solvent.

Figure 3D,G shows that the first three and two components, respectively, of bioactive compounds dataset were positively correlated to biological activities dataset, which allowed for us to say that DIABLO analysis was able to model a good agreement between our datasets. Subsequently, to compare DIABLO models that include/exclude the repeated measures experimental design, we examined the ROC assay (Receiver Operating Characteristic Curve). As we could observe in Figure 3E, the AUC (area under the curve) for the first three component for bioactive compounds and biological activities were 0.89 and 0.77 , respectively. As for the second model, the AUC for the first two component were 1 for both bioactive compounds and biological activities (Figure 3H). Finally, the performance of each model was evaluated by estimating the classification error rate. Centroids distance was used as prediction distance and $10 \times 5$-fold CV as repeated stratified cross-validation. Thus, by observing Figure 3C,F, the best performance was obtained for 3 and 2 component, respectively, which suggests a satisfactory result on our model.

Circos plot and network were carried out to analyze the correlative relationships between total bioactive compound contents of each extract with their biological activities (Figure 4). The analysis revealed that AChE had a positive correlation with total tannin content (TTC) $(r=0.79)$, total saponin content (TSC) $(r=0.78)$ and total flavonol content (TFvLC) $(r=0.86)$, whereas tyrosinase was correlated with TFvLC $(r=0.75)$. Likewise total phenolic content (TPC) and total phenolic acid (TPaC) were positively related to Radical Scavenging Activity ABTS $(r=0.74 ; r=0.77)$ and DPPH $(r=0.74 ; r=0.77)$, Reducing Power ability FRAP $(r=0.77 ; r=0.81)$ and CUPRAC $(r=0.75 ; r=0.78)$ and Ferrous ion Chelation (MCA) $(r=0.73 ; r=0.81)$. Accordingly, it was obvious that the phenolic compounds especially phenolic acid compounds were mostly responsible for the antioxidant activities and metal chelating ability of A. leiocarpus extracts. 
A
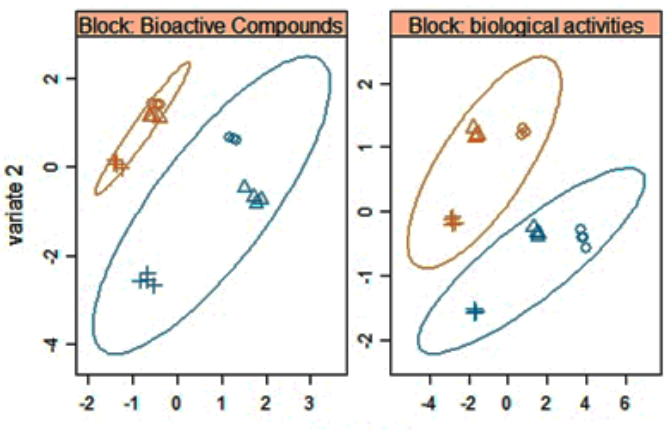

PARTS

Leaves

SOLVENTS

$\triangle \mathrm{MAOH}$

Water

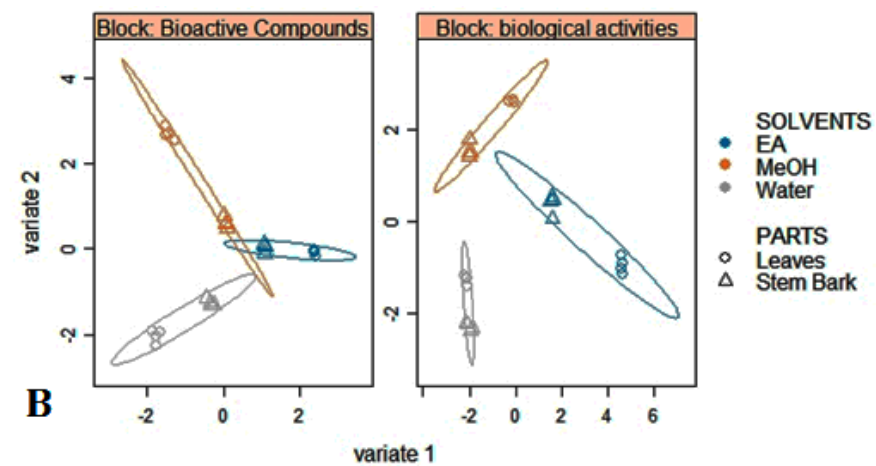

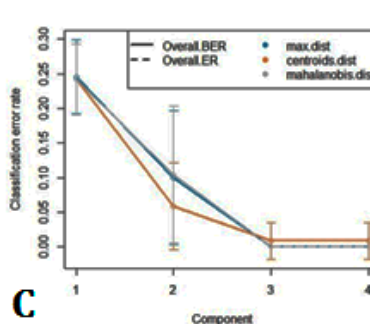

C

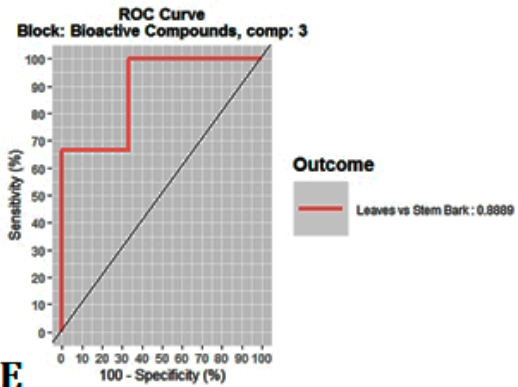

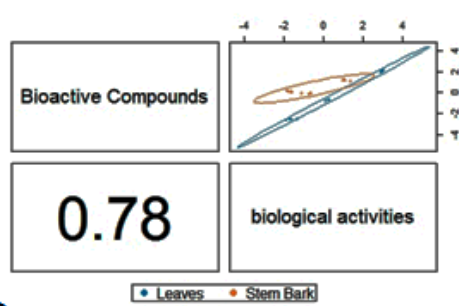
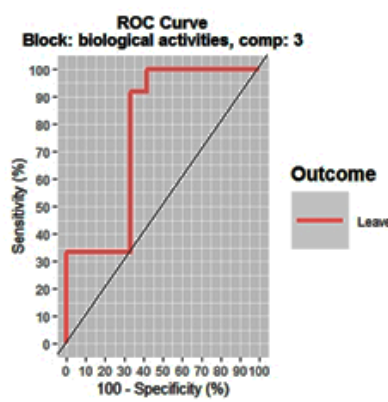

Figure 3. N-integration across multiple datasets analysis on Anogeissus leiocarpus bioactive compounds content and biological activities according to two factors (solvents and parts). (A,B): Sample plot with confidence ellipse according to the parts of plant and the extracting solvent as factor, respectively. (C,F): The model performance per component for Centroids Distance using 5-fold CV repeated 10 times. (D,G): the global overview of the relationship between the two datasets at the two first component level. (E,H): AUC (area under the curve) average and ROC (Receiver Operating Characteristic Curve) curve using one-vs-all comparisons. 

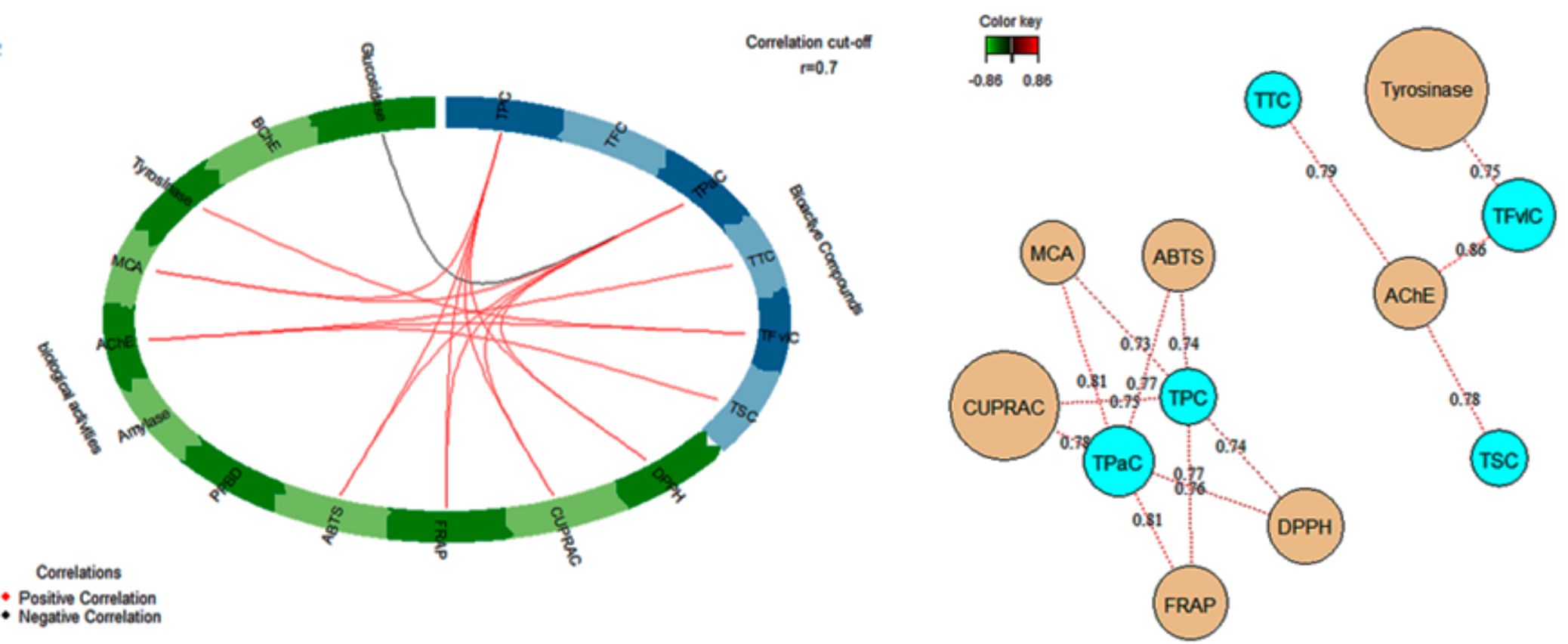

Figure 4. Circos-plot and network showing the relationship between total bioactive compounds and evaluated biological activities (cut-off: $r=0.7$ ). 


\subsection{Pharmacological Studies}

With the aim of investigating extract biological activity, EA, $\mathrm{MeOH}$, and water extracts of A. leiocarpus leaves and stem barks were assayed through the allelopathy test. To this regard, the seeds of the commercial Lollo bionda lettuce cultivar were exposed to scalar extract concentrations $(0.1-10 \mathrm{mg} / \mathrm{mL})$, and the seedling germination and growth were monitored. After incubation of seeds with extracts, we observed a null effect on the seedling germination (Figure 5), thus obtaining a preliminary index of the extract biocompatibility.
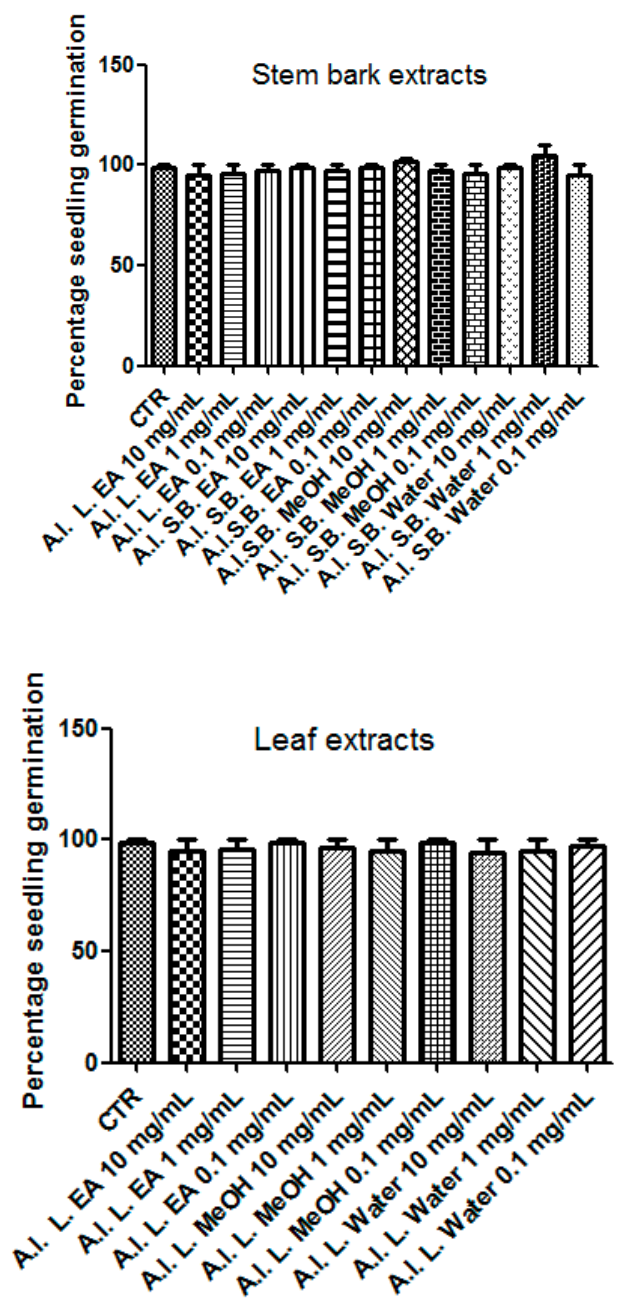

Figure 5. Effects of A. leiocarpus leaf (L) and stem bark (SB) extracts $(0.1-10 \mathrm{mg} / \mathrm{mL})$ on Lollo bionda lettuce root elongation rate. Data, expressed as mean length distribution of germinated seeds, are means \pm SD of three experiments performed in triplicate. After exposing lettuce roots to the extracts, a null effect on seedling germination was observed. EA: Ethyl acetate; MeOH: Methanol.

As a further approach to evaluate potential toxicity, A. leiocarpus extracts, in the concentration range $0.01-10 \mathrm{mg} / \mathrm{mL}$, were tested on brine shrimp lethality assay, performed on the brine shrimp Artemia salina Leach, which is recognized as a valuable tool to predict potential cytotoxicity related to plant extracts [44]. The experimental procedure was conducted in agreement with a previous published paradigm [45]. The results of this assay indicated $\mathrm{LC}_{50}$ values in the range $0.26-2.04 \mathrm{mg} / \mathrm{mL}$ (Figure 6), which were indicatory to choose the extract concentration for the in vitro and ex vivo investigations in order to elucidate putative protective effects, in the colon. 


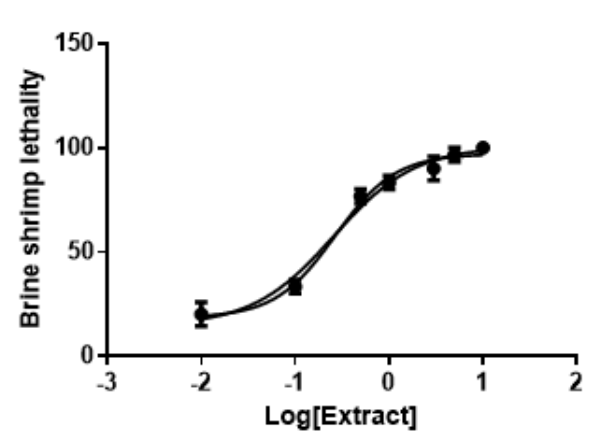

L-EA (LC $50: 0.26 \mathrm{mg} / \mathrm{mL}$ )

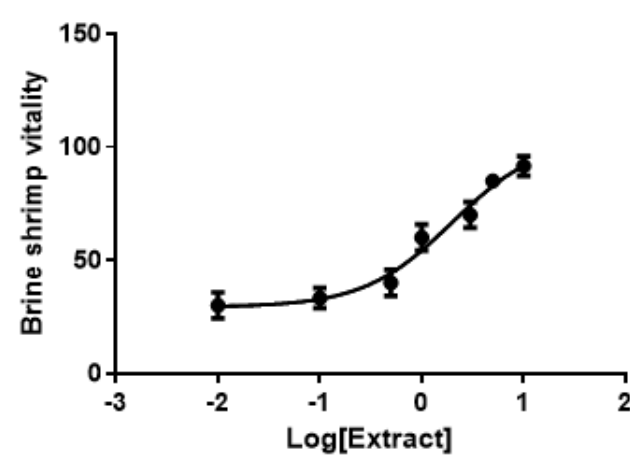

SB-EA (LC $50: 1.98 \mathrm{mg} / \mathrm{mL}$ )

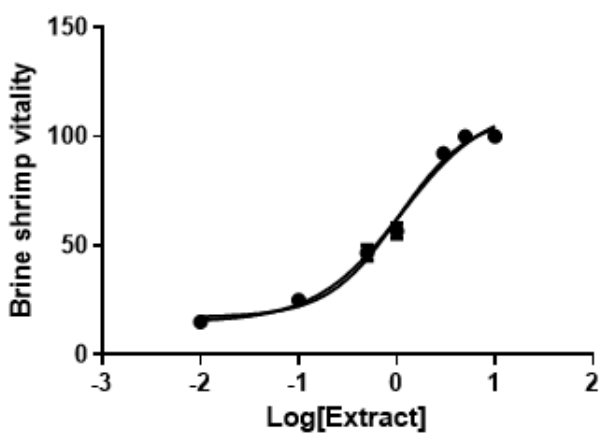

L-MeOH (LC50: 1.04mg/mL)

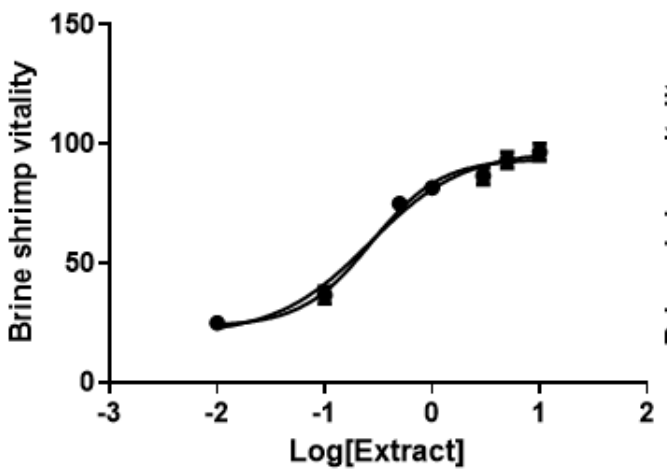

SB-MeOH (LC $50: 0.27 \mathrm{mg} / \mathrm{mL}$ )

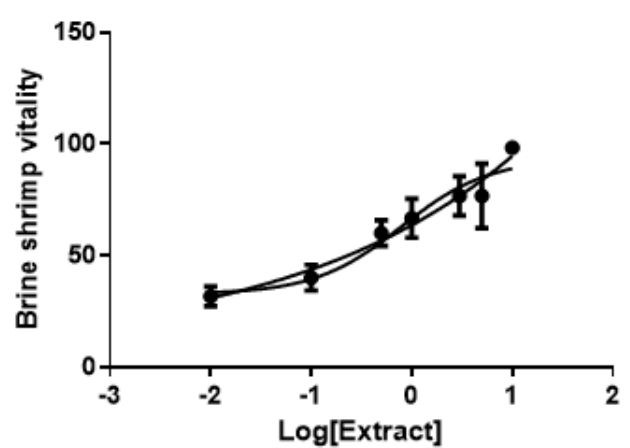

L-Water $\left(\right.$ LC $_{50}: 0.87 \mathrm{mg} / \mathrm{mL}$ )

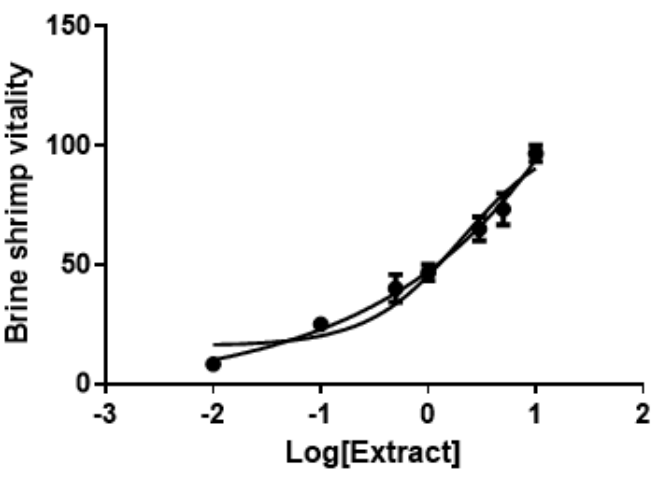

SB-Water ( LC $\left._{50}: 2.04 \mathrm{mg} / \mathrm{mL}\right)$

Figure 6. Effects of A. leiocarpus leaf (L) and stem bark (SB) extracts (0.01-10 mg/mL) on Artemia salina Leach lethality (Brine shrimp lethality test). Data are means \pm $\mathrm{SD}$ of three experiments performed in triplicate. After exposing brine shrimps to the extracts, $\mathrm{LC}_{50}$ values in the range $0.26-2.04 \mathrm{mg} / \mathrm{mL}$ were recorded. EA: Ethyl acetate; $\mathrm{MeOH}$ : Methanol. 
Particularly, we selected the concentration $0.1 \mathrm{mg} / \mathrm{mL}$ that was at least two-fold lower than $\mathrm{LC}_{50}$ and in agreement with previous investigations that demonstrated the antioxidant effects on isolated porcine tissue [46]. While considering these findings, we assayed extract effects on rat colon stimulated with LPS, ex vivo, in order reproduce the burden of oxidative stress and inflammation that characterize ulcerative colitis $[20,47,48]$. All extracts, with the only exception of stembark methanol extract, revealed effective in reducing LPS-induced 8-iso-PGF $2 \alpha$ level (Figure 7). On the other hand, all of the extracts blunted LPS-stimulated PGE 2 colon level (Figure 8), whereas leaf water and stem bark methanol extracts failed to reduce 5-HT concentration (Figure 9). Finally, when the extracts were tested on colon cancer HCT116 cell line, only stem bark ethyl acetate extract revealed biocompatibility, exerting a null effect on cell proliferation (Figures 10 and 11). Conversely, the other extracts displayed stimulatory effects on either viability or spontaneous migration of HCT116 cells.

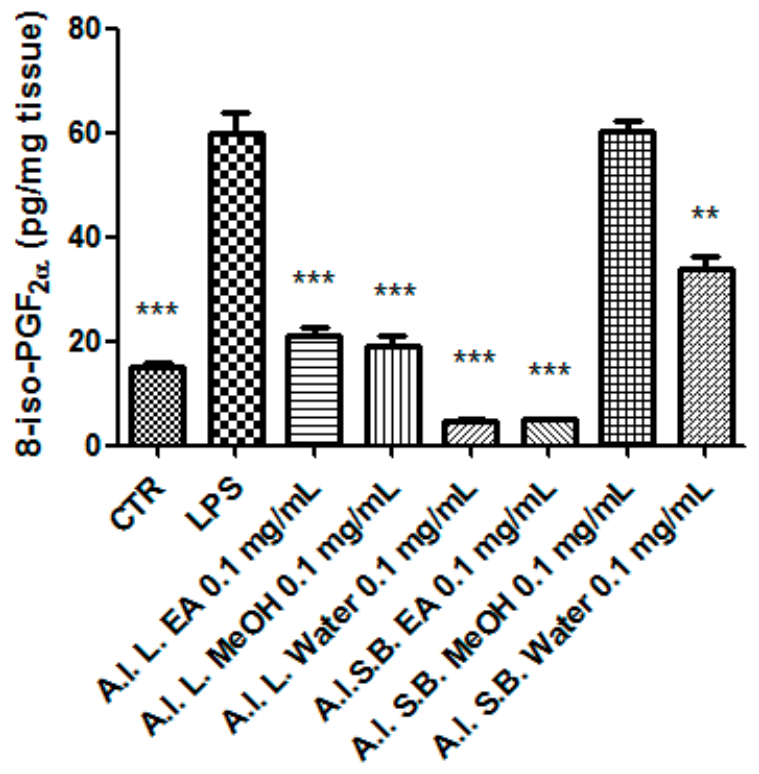

Figure 7. Effect A. leiocarpus leaf (L) and stem bark (SB) extracts $(0.01 \mathrm{mg} / \mathrm{mL})$ on lipopolysaccharide (LPS)-induced 8-iso-prostaglandin(PG) $\mathrm{F}_{2 \alpha}$ level in isolated rat colon. EA: Ethyl acetate; $\mathrm{MeOH}$ : Methanol. ANOVA, $p<0.0001$; post hoc, ${ }^{* *} p<0.01,{ }^{* * *} p<0.001$ vs. LPS.

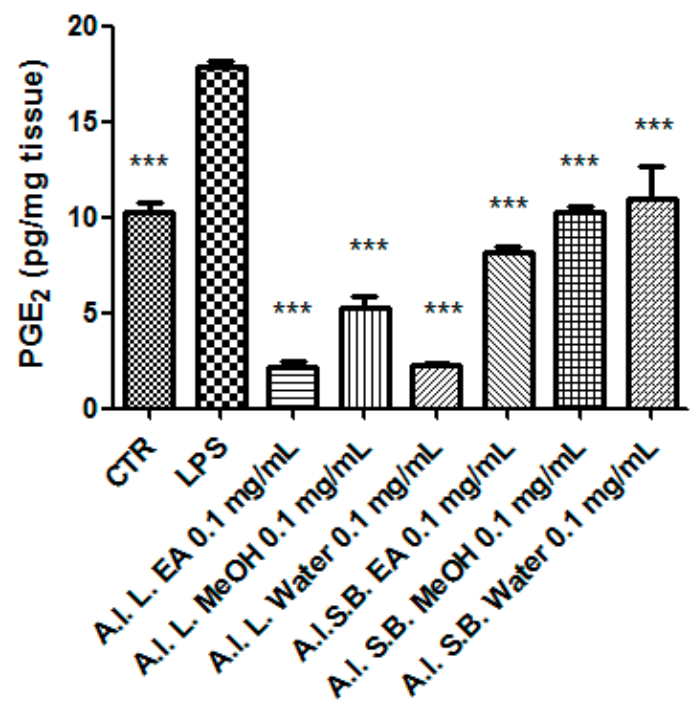

Figure 8. Effect A. leiocarpus leaf (L) and stem bark (SB) extracts $(0.01 \mathrm{mg} / \mathrm{mL})$ on lipopolysaccharide (LPS)-induced prostaglandin(PG) $\mathrm{E}_{2}$ level in isolated rat colon. EA: Ethyl acetate; $\mathrm{MeOH}$ : Methanol. ANOVA, $p<0.0001$; post hoc, ${ }^{* * *} p<0.001$ vs. LPS. 


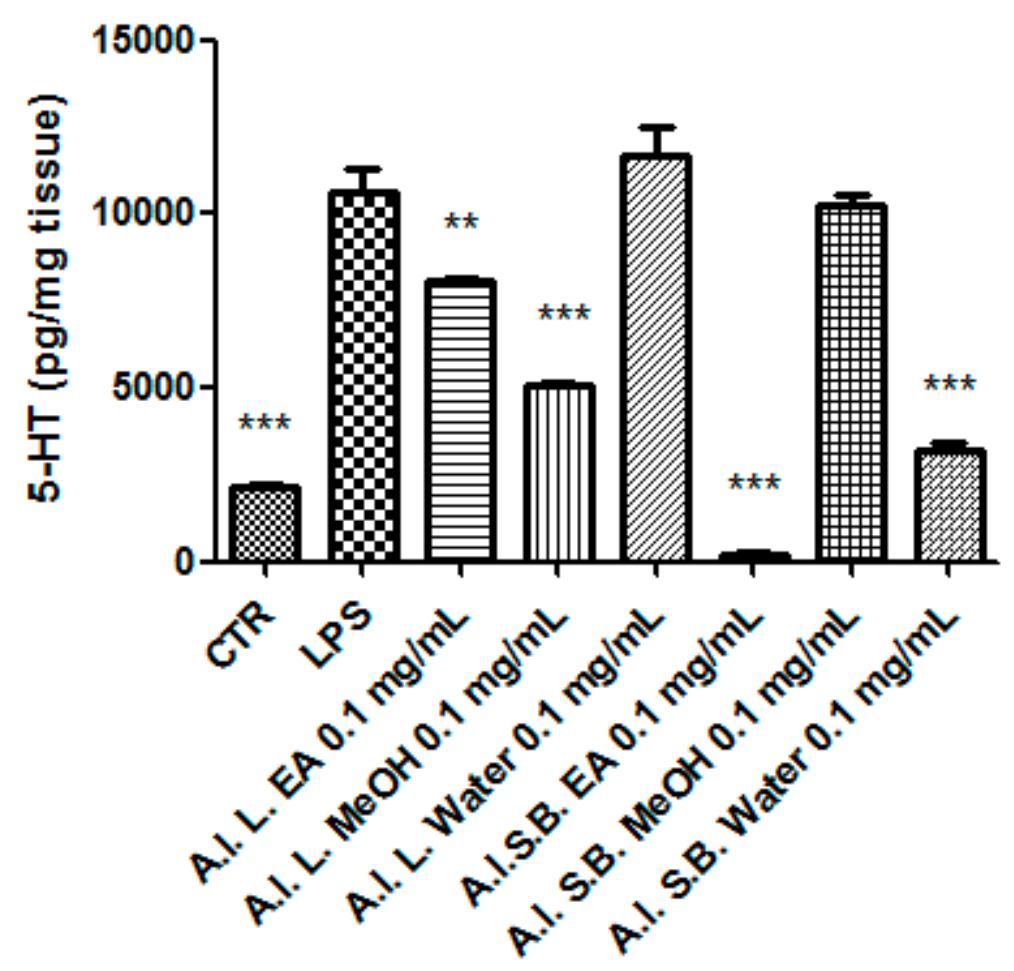

Figure 9. Effect $A$. leiocarpus leaf $(\mathrm{L})$ and stem bark (SB) extracts $(0.01 \mathrm{mg} / \mathrm{mL})$ on lipopolysaccharide (LPS)-induced serotonin (5-HT) level in isolated rat colon. EA: Ethyl acetate; MeOH: Methanol. ANOVA, $p<0.0001$; post hoc, ${ }^{* *} p<0.01,{ }^{* * *} p<0.001$ vs. LPS.

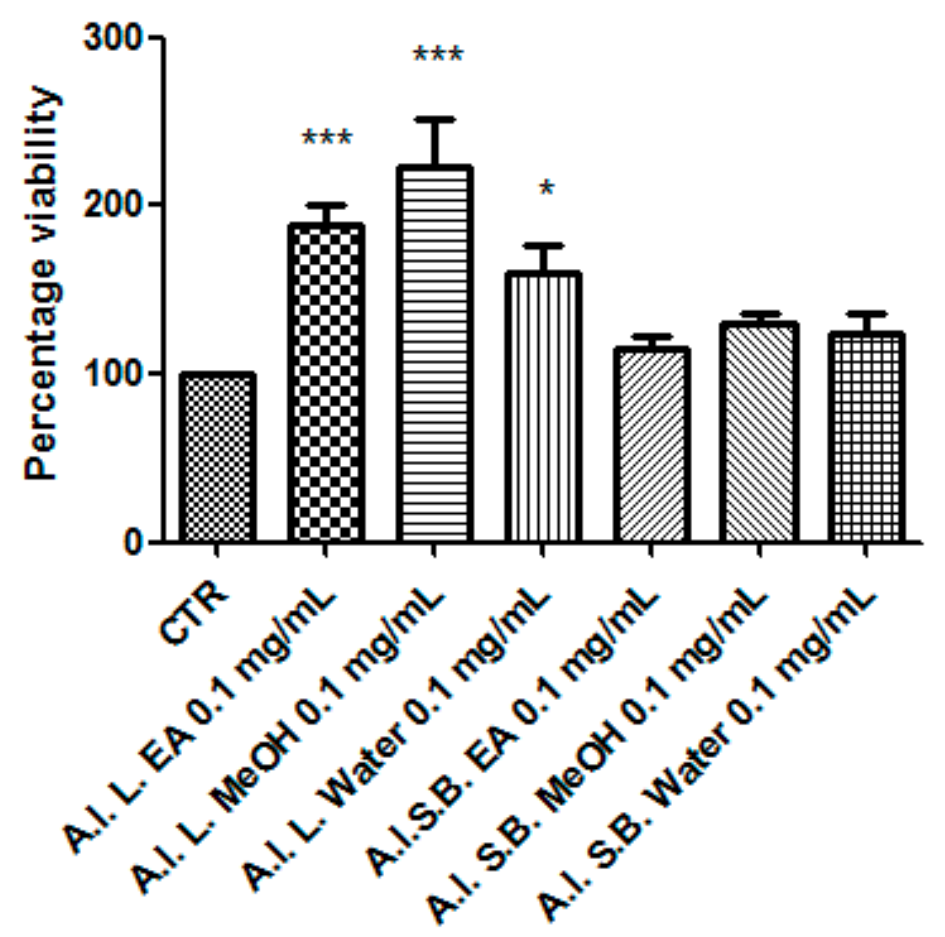

Figure 10. Effect $A$. leiocarpus leaf (L) and stem bark (SB) extracts $(0.01 \mathrm{mg} / \mathrm{mL})$ on human colon cancer HCT116 cell line viability (MTT assay). EA: Ethyl acetate; MeOH: Methanol. ANOVA, $p<0.0001 ;$ post hoc, ${ }^{*} p<0.05,{ }^{* * *} p<0.001$ vs. CTR (Control group). 


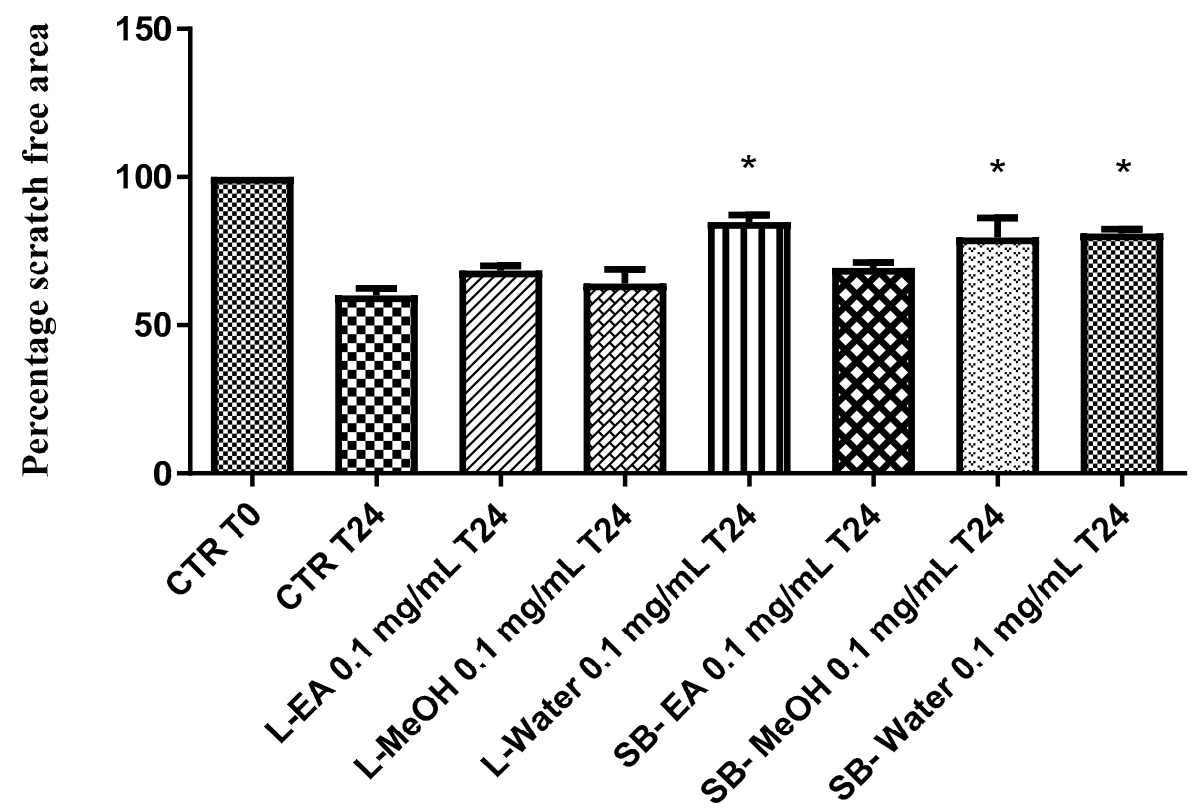

Figure 11. Effect $A$. leiocarpus leaf (L) and stem bark (SB) extracts $(0.01 \mathrm{mg} / \mathrm{mL})$ on human colon cancer HCT116 cell line spontaneous migration (wound healing assay). EA: Ethyl acetate; MeOH: Methanol. ANOVA, $p<0.01$; post hoc, ${ }^{*} p<0.05$ vs. CTR T24 (Control group).

\section{Discussion}

Oxidative stress is characterized by the overproduction of reactive oxygen/nitrogen (ROS/RNS) species that could drive to lipid peroxidation [49], which displays a key role in the pathogenesis of ulcerative colitis [50]. 8-iso- $\mathrm{PGF}_{2 \alpha}$, deriving from ROS/RNS peroxidation of membrane arachidonic acid, represents a stable marker of lipid peroxidation and tissue damage, in vivo [51], whereas the blunting effects on 8 -iso- $\mathrm{PGF}_{2 \alpha}$ production that are induced by herbal extracts were related to protective effects [48,52]. Consistently with the reported antioxidant effects and the findings by Belemnaba et al. [46], the tested extracts blunted LPS-induced 8-iso- $\mathrm{PGF}_{2 \alpha}$ production, with the only exception being represented by stem bark $\mathrm{MeOH}$ extract. On the other hand, stem bark $\mathrm{MeOH}$ extract showed the ability to reduce the level of malondialdehyde (MDA), which is another key marker of lipid peroxidation, in vivo [53]. This discrepancy could depend on more than one speculation. On one side, the differences in the employed experimental ex vivo and in vivo paradigms that were chosen by us and Akanbi and colleagues [53], respectively, could be crucial. On the other side, we should also consider that isoprostanes derive only by arachidonic acid peroxidation, whereas MDA could originate from various polyunsaturated acids [54], and this could represent a limit in the evaluation of lipid peroxidation.

The effects of A. leiocarpus extracts on LPS-induced levels of colon $\mathrm{PGE}_{2}$ were investigated as well. $\mathrm{PGE}_{2}$ is a cyclooxygenase (COX)-2-derived pro-inflammatory mediator, whose upregulation has been long involved in colon inflammation and damage, whereas the antioxidants were revealed to be effective in blunting the colon levels of this prostaglandin [47,55]. All of the tested extracts proved able in down-regulating LPS-induced $\mathrm{PGE}_{2}$ level (Figure 8), consistently with the reported antioxidant effects.

A blunting effect on LPS-induced 5-HT level was observed after treating the colon specimens with A. leiocarpus extract, as well. 5-HT pro-inflammatory role in ulcerative colitis was previously suggested [56], which possibly involved the activation of 5- $\mathrm{HT}_{3}$ receptors [57]. With the only exception of leaf water and stem bark $\mathrm{MeOH}$ extracts, the A. leiocarpus extracts displayed a significant inhibition of 5 -HT steady state level, in the colon. This could be, albeit partially, related to decreased neurotransmitter synthesis and release, in the colon tissue [58,59]. 
Collectively, all of the tested A. leiocarpus extracts could play a noteworthy anti-inflammatory role, as indicated by their blunting effects on LPS-stimulated PGE 2 level. On the other hand, the lack of efficacy that was exerted by the leaf water and stem bark MeOH extracts on LPS-induced levels of 5-HT and 8-iso-PGF $2 \alpha$ suggests that the lower quantitative profile of gallic acid, catechin, and epicatechin could limit the antioxidant potency, as compared to the other tested extracts.

Finally, the extracts were tested for their putative anti-proliferative role against the human colon HCT116 cell line, which was previously found to be sensitive to different polarity extracts from A. latifolia [12]. In the present study, the anti-proliferative effects were investigated through validated in vitro tests, including MTT and wound healing assays. A different pattern of effects on cell viability was observed after exposing HCT116 cells to A. leiocarpus extracts. On one side, stem bark extracts displayed a null effect on cell viability (Figure 10), which resulted in the range of biocompatibility ( $>70 \%$ and $<130 \%$ as compared to control group). On the other side, leaf extract increased significantly HCT116 cell viability ( $>140 \%$ as compared to control group). Actually, the stimulating effect on HCT116 cell viability induced by leaf extracts could be related to the their higher qualitative content of metabolites that are related to quercetin, which was found to exert protective effects on this cell line [60]. Additionally, water leaf and water and MeOH stem bark extracts induced spontaneous HCT116 cell migration in wound healing assay (Figure 11), thus also indicating a potential stimulating-effect on invasion capacity. Conversely, stem bark EA and leaf EA and MeOH extracts did not exert any influence on spontaneous HCT116 cell migration, in the $24 \mathrm{~h}$ following experimental lesion induced on cell monolayer. Actually, the different effects that were showed by the tested extracts in the wound healing paradigm could be related, at least in part, to the different content of gallic acid, which was found to inhibit spontaneous cell migration [61]. As a conclusive note of the pharmacological investigation, it resulted of particular interest the antioxidant/anti-inflammatory profile that was exerted by the stem bark EA extract, together with its null effect on colon cancer cell proliferation. The highest inhibitory effect that is exerted by this extract on colon 5-HT level could be one of the main causes leading to the null effect on HCT116 viability and spontaneous migration [62].

\section{Conclusions}

Data that are presented in this study highlighted the key role of solvent choice in the quest for novel bioactive compounds from plants. It was demonstrated that water and methanol were good solvents for the extraction of phytochemicals having antioxidant properties. The methanol extract of A. leiocarpus leaves was also an active cholinesterase inhibitor, while the methanol extract of the stem bark inhibited tyrosinase. On the other hand, the ethyl acetate extract of $A$. leiocarpus leaves and stem bark showed potent inhibition against $\alpha$-glucosidase. The pharmacological study that was carried out on isolated colon and HCT116 cell line further deepened the spectrum of potential application of the present extracts. Noteworthy interest derives from the stem bark EA extract that, besides exerting the best antioxidant/anti-inflammatory profile, was the only one that was unable to stimulate the proliferation of human colon cancer HCT116 cell line, thus supporting potential application in the prevention of the oxidative stress-induced tissue damage occurring in ulcerative colitis. As a conclusion, A. leiocarpus stem bark EA extract represents a potential source of bioactive compounds for the development of novel therapeutic agents.

Supplementary Materials: The following are available online at http://www.mdpi.com/2076-3921/8/9/343/s1. Table S1: Chemical composition of A. leiocarpus leaves ethyl acetate extract. Table S2: Chemical composition of A. leiocarpus leaves methanol extract. Table S3: Chemical composition of A. leiocarpus leaves water extract. Table S4: Chemical composition of A. leiocarpus stem bark ethyl acetate extract. Table S5: Chemical composition of A. leiocarpus stem bark methanol extract. Table S6: Chemical composition of A. leiocarpus stem bark water extract.

Author Contributions: Conceptualization, C.F. and G.Z.; methodology, G.O.; L.M.; software, L.M.; validation, C.F.; G.Z.; L.M.; G.O.; formal analysis, C.F.; G.Z.; investigation, K.I.S.; K.B.; A.D.; J.J.; L.R.; A.C.; S.L.; Z.C.; S.D.S.; C.M.N.P.-A.; resources, C.F.; G.O.; L.M.; data curation, C.F.; G.Z.; writing-original draft preparation, M.F.M.; writing-review and editing, C.F., G.Z.; G.O.; L.M.; visualization, L.B.; supervision, L.B.; project administration, C.F.; L.M.; G.O.; G.Z.; funding acquisition, C.F.; G.O.; L.M. 
Acknowledgments: This work was supported by grants from the Italian Ministry of University (FAR 2016 granted to Claudio Ferrante; FAR 2018 granted to Giustino Orlando; FAR 2016 granted to Luigi Menghini).

Conflicts of Interest: The authors declare no conflict of interest.

\section{References}

1. Chaabi, M.; Benayache, S.; Benayache, F.; N'Gom, S.; Koné, M.; Anton, R.; Weniger, B.; Lobstein, A. Triterpenes and polyphenols from Anogeissus leiocarpus (Combretaceae). Biochem. Syst. Ecol. 2008, 36, 59-62. [CrossRef]

2. Salih, E.Y.A.; Kanninen, M.; Sipi, M.; Luukkanen, O.; Hiltunen, R.; Vuorela, H.; Julkunen-Tiitto, R.; Fyhrquist, P. Tannins, flavonoids and stilbenes in extracts of African savanna woodland trees Terminalia brownii, Terminalia laxiflora and Anogeissus leiocarpus showing promising antibacterial potential. S. Afr. J. Bot. 2017, 108, 370-386. [CrossRef]

3. Ademosun, A.O.; Adebayo, A.A.; Oboh, G. Anogeissus leiocarpus attenuates paroxetine-induced erectile dysfunction in male rats via enhanced sexual behavior, nitric oxide level and antioxidant status. Biomed. Pharmacother. 2019, 111, 1029-1035. [CrossRef] [PubMed]

4. Etuk, E.; Mohammed, B. Informant consensus selection method: A reliability assessment on medicinal plants used in north western Nigeria for the treatment of diabetes mellitus. Afr. J. Pharm. Pharmacol. 2009, 3, 496-500.

5. Adefegha, S.A.; Oboh, G.; Omojokun, O.S.; Jimoh, T.O.; Oyeleye, S.I. In vitro antioxidant activities of African birch (Anogeissus leiocarpus) leaf and its effect on the $\alpha$-amylase and $\alpha$-glucosidase inhibitory properties of acarbose. J. Taibah Univ. Med. Sci. 2016, 11, 236-242. [CrossRef]

6. Belemnaba, L.; Nitiema, M.; Ilboudo, S.; Ouedraogo, N.; Ouedraogo, G.G.; Belemlilga, M.B.; Ouedraogo, S.; Guissou, I.P. O8 Study on antihypertensive activity of an aqueous extract of Anogeissus leiocarpus (AEAL) DC Guill et Perr bark of trunk in L-NAME-induced hypertensive rats. Biochem. Pharmacol. 2017, 139, 112. [CrossRef]

7. Victor, B.Y.; Grace, A. Phytochemical studies, in-vitro antibacterial activities and antioxidant properties of the methanolic and ethyl acetate extracts of the leaves of Anogeissus leiocarpus. Int. J. Biochem. Res. Rev. 2013, 3, 137. [CrossRef]

8. Shuaibu, M.N.; Wuyep, P.T.; Yanagi, T.; Hirayama, K.; Ichinose, A.; Tanaka, T.; Kouno, I. Trypanocidal activity of extracts and compounds from the stem bark of Anogeissus leiocarpus and Terminalia avicennoides. Parasitol. Res. 2008, 102, 697-703. [CrossRef]

9. Shuaibu, M.N.; Pandey, K.; Wuyep, P.A.; Yanagi, T.; Hirayama, K.; Ichinose, A.; Tanaka, T.; Kouno, I. Castalagin from Anogeissus leiocarpus mediates the killing of Leishmania in vitro. Parasitol. Res. 2008, 103, 1333-1338. [CrossRef]

10. Vonthron-Sénécheau, C.; Weniger, B.; Ouattara, M.; Bi, F.T.; Kamenan, A.; Lobstein, A.; Brun, R.; Anton, R. In vitro antiplasmodial activity and cytotoxicity of ethnobotanically selected Ivorian plants. J. Ethnopharmacol. 2003, 87, 221-225. [CrossRef]

11. Akanbi, O.M.; Omonkhua, A.A.; Cyril-Olutayo, C.M. Effect of crude methanolic extract of Anogeissus leiocarpus on the liver function of P. berghei infected mice. Int. J. Infect. Dis. 2014, 21, 196. [CrossRef]

12. Diab, K.A.; Guru, S.K.; Bhushan, S.; Saxena, A.K. In vitro anticancer activities of Anogeissus latifolia, Terminalia bellerica, Acacia catechu and Moringa oleiferna Indian plants. Asian Pac. J. Cancer Prev. 2015, 16, 6423-6428. [CrossRef] [PubMed]

13. Ferrante, C.; Recinella, L.; Ronci, M.; Menghini, L.; Brunetti, L.; Chiavaroli, A.; Leone, S.; Di Iorio, L.; Carradori, S.; Tirillini, B.; et al. Multiple pharmacognostic characterization on hemp commercial cultivars: Focus on inflorescence water extract activity. Food Chem. Toxicol. 2019, 125, 452-461. [CrossRef] [PubMed]

14. Zengin, G.; Aktumsek, A. Investigation of antioxidant potentials of solvent extracts from different anatomical parts of Asphodeline anatolica E. Tuzlaci: An endemic plant to Turkey. Afr. J. Tradit. Complement. Altern. Med. 2014, 11, 481-488. [CrossRef] [PubMed]

15. Zengin, G.; Llorent-Martínez, E.J.; Fernández-de Córdova, M.L.; Bahadori, M.B.; Mocan, A.; Locatelli, M.; Aktumsek, A. Chemical composition and biological activities of extracts from three Salvia species: S. blepharochlaena, S. euphratica var. leiocalycina, and S. verticillata subsp. amasiaca. Ind. Crops Prod. 2018, 111, 11-21. [CrossRef] 
16. Zengin, G.; Uysal, A.; Diuzheva, A.; Gunes, E.; Jekő, J.; Cziáky, Z.; Picot-Allain, C.M.N.; Mahomoodally, M.F. Characterization of phytochemical components of Ferula halophila extracts using HPLC-MS/MS and their pharmacological potentials: A multi-functional insight. J. Pharm. Biomed. Anal. 2018, 160,374-382. [CrossRef] [PubMed]

17. Rodriguez-Delgado, M.; Malovana, S.; Perez, J.; Borges, T.; Montelongo, F.G. Separation of phenolic compounds by high-performance liquid chromatography with absorbance and fluorimetric detection. $J$. Chromatogr. A 2001, 912, 249-257. [CrossRef]

18. Uysal, S.; Zengin, G.; Locatelli, M.; Bahadori, M.B.; Mocan, A.; Bellagamba, G.; De Luca, E.; Mollica, A.; Aktumsek, A. Cytotoxic and enzyme inhibitory potential of two Potentilla species (P. speciosa L. and P. reptans Willd.) and their chemical composition. Front. Pharmacol. 2017, 8, 290. [CrossRef]

19. Mahmoodzadeh, H.; GhasemI, M.; Zanganeh, H. Allelopathic effect of medicinal plant Cannabis sativa L. on Lactuca sativa L. seed germination. Acta Agric. Slov. 2015, 105, 233-239. [CrossRef]

20. Brunetti, L.; Orlando, G.; Ferrante, C.; Recinella, L.; Leone, S.; Chiavaroli, A.; Di Nisio, C.; Shohreh, R.; Manippa, F.; Ricciuti, A.; et al. Peripheral chemerin administration modulates hypothalamic control of feeding. Peptides 2014, 51, 115-121. [CrossRef]

21. Ferrante, C.; Orlando, G.; Recinella, L.; Leone, S.; Chiavaroli, A.; Di Nisio, C.; Shohreh, R.; Manippa, F.; Ricciuti, A.; Vacca, M.; et al. Central inhibitory effects on feeding induced by the adipo-myokine irisin. Eur. J. Pharmacol. 2016, 791, 389-394. [CrossRef] [PubMed]

22. Chiavaroli, A.; Brunetti, L.; Orlando, G.; Recinella, L.; Ferrante, C.; Leone, S.; Di Michele, P.; Di Nisio, C.; Vacca, M. Resveratrol inhibits isoprostane production in young and aged rat brain. J. Biol. Regul. Homeost. Agents 2010, 24, 441. [PubMed]

23. Locatelli, M.; Macchione, N.; Ferrante, C.; Chiavaroli, A.; Recinella, L.; Carradori, S.; Zengin, G.; Cesa, S.; Leporini, L.; Leone, S. Graminex pollen: Phenolic pattern, colorimetric analysis and protective effects in immortalized prostate cells (PC3) and rat prostate challenged with LPS. Molecules 2018, 23, 1145. [CrossRef] [PubMed]

24. National Centre for Replacement Refinement\& Reduction of Animals in Research. Available online: https://www.nc3rs.org.uk/experimental-designstatistics (accessed on 16 August 2019).

25. Galanakis, C.M.; Goulas, V.; Tsakona, S.; Manganaris, G.A.; Gekas, V. A Knowledge Base for the Recovery of Natural Phenols with Different Solvents. Int. J. Food Prop. 2013, 16, 382-396. [CrossRef]

26. Ji, W.; Meng, Q.; Ding, L.; Wang, F.; Dong, J.; Zhou, G.; Wang, B. Measurement and correlation of the solubility of caffeic acid in eight mono and water+ethanol mixed solvents at temperatures from (293.15 to 333.15) K. J. Mol. Liq. 2016, 224, 1275-1281. [CrossRef]

27. Lin, L.-T.; Chen, T.-Y.; Chung, C.-Y.; Noyce, R.S.; Grindley, T.B.; McCormick, C.; Lin, T.-C.; Wang, G.-H.; Lin, C.-C.; Richardson, C.D. Hydrolyzable Tannins (Chebulagic Acid and Punicalagin) Target Viral Glycoprotein-Glycosaminoglycan Interactions To Inhibit Herpes Simplex Virus 1 Entry and Cell-to-Cell Spread. J. Virol. 2011, 85, 4386-4398. [CrossRef] [PubMed]

28. Perera, H.D.S.M.; Samarasekera, J.K.R.R.; Handunnetti, S.M.; Weerasena, O.V.D.S.J. In vitro anti-inflammatory and anti-oxidant activities of Sri Lankan medicinal plants. Ind. Crops Prod. 2016, 94, 610-620. [CrossRef]

29. Holtz, R.W. Chapter 13-In Vitro Methods to Screen Materials for Anti-aging Effects. In Skin Aging Handbook; Dayan, N., Ed.; William Andrew Publishing: Norwich, NY, USA, 2009; pp. 329-362.

30. Cerretani, L.; Bendini, A. Chapter 67-Rapid Assays to Evaluate the Antioxidant Capacity of Phenols in Virgin Olive Oil. In Olives and Olive Oil in Health and Disease Prevention; Preedy, V.R., Watson, R.R., Eds.; Academic Press: San Diego, CA, USA, 2010; pp. 625-635.

31. Özyürek, M.; Güçlü, K.; Tütem, E.; Başkan, K.S.; Erçă̆, E.; Celik, S.E.; Baki, S.; Yıldız, L.; Karaman, Ş.; Apak, R. A comprehensive review of CUPRAC methodology. Anal. Methods 2011, 3, 2439-2453. [CrossRef]

32. Puntarulo, S. Iron, oxidative stress and human health. Mol. Asp. Med. 2005, 26, 299-312. [CrossRef]

33. Mira, L.; Fernandez, M.T.; Santos, M.; Rocha, R.; Florencio, M.H.; Jennings, K.R. Interactions of flavonoids with iron and copper ions: A mechanism for their antioxidant activity. Free Radic. Res. 2002, 36, 1199-1208. [CrossRef]

34. Mandel, S.; Amit, T.; Reznichenko, L.; Weinreb, O.; Youdim, M.B. Green tea catechins as brain-permeable, natural iron chelators-antioxidants for the treatment of neurodegenerative disorders. Mol. Nutr. Food Res. 2006, 50, 229-234. [CrossRef] [PubMed] 
35. Liu, Y.; Guo, M. Studies on Transition Metal-Quercetin Complexes Using Electrospray Ionization Tandem Mass Spectrometry. Molecules 2015, 20, 8583-8594. [CrossRef] [PubMed]

36. Santos, M.A.; Chand, K.; Chaves, S. Recent progress in multifunctional metal chelators as potential drugs for Alzheimer's disease. Coord. Chem. Rev. 2016, 327, 287-303. [CrossRef]

37. Ragab, H.M.; Teleb, M.; Haidar, H.R.; Gouda, N. Chlorinated tacrine analogs: Design, synthesis and biological evaluation of their anti-cholinesterase activity as potential treatment for Alzheimer's disease. Bioorg. Chem. 2019, 86, 557-568. [CrossRef] [PubMed]

38. de Andrade, P.; Mantoani, S.P.; Gonçalves Nunes, P.S.; Magadán, C.R.; Pérez, C.; Xavier, D.J.; Hojo, E.T.S.; Campillo, N.E.; Martínez, A.; Carvalho, I. Highly potent and selective aryl-1,2,3-triazolyl benzylpiperidine inhibitors toward butyrylcholinesterase in Alzheimer's disease. Bioorg. Med. Chem. 2019, 27, 931-943. [CrossRef] [PubMed]

39. Liyanaarachchi, G.D.; Samarasekera, J.K.R.R.; Mahanama, K.R.R.; Hemalal, K.D.P. Tyrosinase, elastase, hyaluronidase, inhibitory and antioxidant activity of Sri Lankan medicinal plants for novel cosmeceuticals. Ind. Crops Prod. 2018, 111, 597-605. [CrossRef]

40. Wang, R.; Wang, G.; Xia, Y.; Sui, W.; Si, C. Functionality study of lignin as a tyrosinase inhibitor: Influence of lignin heterogeneity on anti-tyrosinase activity. Int. J. Biol. Macromol. 2019, 128, 107-113. [CrossRef]

41. Chen, Y.H.; Huang, L.; Wen, Z.H.; Zhang, C.; Liang, C.H.; Lai, S.T.; Luo, L.Z.; Wang, Y.Y.; Wang, G.H. Skin whitening capability of shikimic acid pathway compound. Eur. Rev. Med. Pharmacol. Sci. 2016, 20, 1214-1220.

42. Mapunya, M.B.; Hussein, A.A.; Rodriguez, B.; Lall, N. Tyrosinase activity of Greyia flanaganii (Bolus) constituents. Phytomedicine 2011, 18, 1006-1012. [CrossRef]

43. Yao, Y.; Cheng, X.; Wang, L.; Wang, S.; Ren, G. Mushroom tyrosinase inhibitors from mung bean (Vigna radiatae L.) extracts. Int. J. Food Sci. Nutr. 2012, 63, 358-361. [CrossRef]

44. Ohikhena, F.U.; Wintola, O.A.; Afolayan, A.J. Toxicity Assessment of Different Solvent Extracts of the Medicinal Plant, Phragmanthera capitata (Sprengel) Balle on Brine Shrimp (Artemia salina). Int. J. Pharmacol. 2016, 12, 701-710.

45. Taviano, M.F.; Marino, A.; Trovato, A.; Bellinghieri, V.; Melchini, A.; Dugo, P.; Cacciola, F.; Donato, P.; Mondello, L.; Güvenç, A. Juniperus oxycedrus L. subsp. oxycedrus and Juniperus oxycedrus L. subsp. macrocarpa (Sibth. \& Sm.) Ball. "berries" from Turkey: Comparative evaluation of phenolic profile, antioxidant, cytotoxic and antimicrobial activities. Food Chem. Toxicol. 2013, 58, 22-29. [PubMed]

46. Belemnaba, L.; Ouédraogo, S.; Nitiéma, M.; Chataigneau, T.; Guissou, I.P.; Schini-Kerth, V.B.; Bucher, B.; Auger, C. An aqueous extract of the Anogeissus leiocarpus bark (AEAL) induces the endothelium-dependent relaxation of porcine coronary artery rings involving predominantly nitric oxide. J. Basic Clin. Physiol. Pharmacol. 2018, 29, 599-608. [CrossRef] [PubMed]

47. Locatelli, M.; Ferrante, C.; Carradori, S.; Secci, D.; Leporini, L.; Chiavaroli, A.; Leone, S.; Recinella, L.; Orlando, G.; Martinotti, S. Optimization of aqueous extraction and biological activity of Harpagophytum procumbens root on ex vivo rat colon inflammatory model. Phytother. Res. 2017, 31, 937-944. [CrossRef] [PubMed]

48. Menghini, L.; Ferrante, C.; Leporini, L.; Recinella, L.; Chiavaroli, A.; Leone, S.; Pintore, G.; Vacca, M.; Orlando, G.; Brunetti, L. An hydroalcoholic chamomile extract modulates inflammatory and immune response in HT29 cells and isolated rat colon. Phytother. Res. 2016, 30, 1513-1518. [CrossRef]

49. Uttara, B.; Singh, A.V.; Zamboni, P.; Mahajan, R. Oxidative stress and neurodegenerative diseases: A review of upstream and downstream antioxidant therapeutic options. Curr. Neuropharmacol. 2009, 7, 65-74. [CrossRef] [PubMed]

50. Achitei, D.; Ciobica, A.; Balan, G.; Gologan, E.; Stanciu, C.; Stefanescu, G. Different profile of peripheral antioxidant enzymes and lipid peroxidation in active and non-active inflammatory bowel disease patients. Dig. Dis. Sci. 2013, 58, 1244-1249. [CrossRef] [PubMed]

51. Praticò, D. Alzheimer's disease and oxygen radicals: New insights. Biochem. Pharmacol. 2002, 63, 563-567. [CrossRef]

52. Brunetti, L.; Menghini, L.; Orlando, G.; Recinella, L.; Leone, S.; Epifano, F.; Lazzarin, F.; Chiavaroli, A.; Ferrante, C.; Vacca, M. Antioxidant effects of garlic in young and aged rat brain in vitro. J. Med. Food 2009, 12, 1166-1169. [CrossRef] 
53. Akanbi, O.M.; Omonkhua, A.A.; Cyril-Olutayo, C.M.; Fasimoye, R.Y. The antiplasmodial activity of Anogeissus leiocarpus and its effect on oxidative stress and lipid profile in mice infected with Plasmodium bergheii. Parasitol. Res. 2012, 110, 219-226. [CrossRef]

54. Tsikas, D. Assessment of lipid peroxidation by measuring malondialdehyde (MDA) and relatives in biological samples: Analytical and biological challenges. Anal. Biochem. 2017, 524, 13-30. [CrossRef] [PubMed]

55. Yu, L.; Yan, J.; Sun, Z. D-limonene exhibits anti-inflammatory and antioxidant properties in an ulcerative colitis rat model via regulation of iNOS, COX-2, PGE2 and ERK signaling pathways. Mol. Med. Rep. 2017, 15, 2339-2346. [CrossRef] [PubMed]

56. Regmi, S.C.; Park, S.-Y.; Ku, S.K.; Kim, J.-A. Serotonin regulates innate immune responses of colon epithelial cells through Nox2-derived reactive oxygen species. Free Radic. Biol. Med. 2014, 69, 377-389. [CrossRef] [PubMed]

57. Mousavizadeh, K.; Rahimian, R.; Fakhfouri, G.; Aslani, F.; Ghafourifar, P. Anti-inflammatory effects of 5-HT3 receptor antagonist, tropisetron on experimental colitis in rats. Eur. J. Clin. Investig. 2009, 39, 375-383. [CrossRef] [PubMed]

58. Bungo, T.; Shiraishi, J.-i.; Yanagita, K.; Ohta, Y.; Fujita, M. Effect of nociceptin/orphanin FQ on feeding behavior and hypothalamic neuropeptide expression in layer-type chicks. Gen. Comp. Endocrinol. 2009, 163, 47-51. [CrossRef]

59. Clark, K.A.; MohanKumar, S.M.; Kasturi, B.S.; MohanKumar, P.S. Effects of central and systemic administration of leptin on neurotransmitter concentrations in specific areas of the hypothalamus. Am. J. Physiol. Regul. Integr. Comp. Physiol. 2006, 290, R306-R312. [CrossRef] [PubMed]

60. Salem, I.B.; Boussabbeh, M.; Graiet, I.; Rhouma, A.; Bacha, H.; Essefi, S.A. Quercetin protects HCT116 cells from Dichlorvos-induced oxidative stress and apoptosis. Cell Stress Chaperones 2016, 21, 179-186. [CrossRef] [PubMed]

61. Wang, X.; Liu, K.; Ruan, M.; Yang, J.; Gao, Z. Gallic acid inhibits fibroblast growth and migration in keloids through the AKT/ERK signaling pathway. Acta Biochim. Biophys. Sin. 2018, 50, 1114-1120. [CrossRef]

62. Sui, X.; Zhu, J.; Tang, H.; Wang, C.; Zhou, J.; Han, W.; Wang, X.; Fang, Y.; Xu, Y.; Li, D.; et al. p53 controls colorectal cancer cell invasion by inhibiting the NF-кB-mediated activation of Fascin. Oncotarget 2015, 6, 22869-22879. [CrossRef] 